\title{
Second Harmonic Generation Signal from Biological Materials Using Multi-Functional Two-Photon Laser Scanning Microscopy
}

\author{
Ali Hussain Reshak \\ Institute of Physical Biology-South Bohemia University, \\ Nove Hrady 37333 \\ ${ }_{2}^{2}$ School of Microelectronic Engineering, University Malaysia Perlis \\ (UniMAP), Block A, Kompleks Pusat Pengajian, 02600 Arau ckjawi, Perlis \\ ${ }^{1}$ Czech Republic \\ ${ }^{2}$ Malaysia
}

\section{Introduction}

The nonlinear optical effect, such as two-photon (Denk et al., 1990) and three-photon (Maiti et al., 1997; Schrader et al., 1997; Wokosin et al., 1996) fluorescence significantly improved the depth resolution and reduced the background noise. The nonlinear optical techniques have been used to develop a new generation of optical microscopes with novel capabilities. These new capabilities include the ability of using near-infrared light to induce absorption, and hence fluorescence from fluorophores that are absorbed in the ultraviolet region. Other capabilities of nonlinear microscopes include: improved spatial and temporal resolution without the use of pinholes or slits for spatial filtering, improved signal strength, deeper penetration into thick, highly scattering tissue and confinement of photo-bleaching to the focal volume (Denk et al. 1990). The invention of nonlinear laser microscopy opened new opportunity to noninvasively examine the structure and function of living cells or tissues (Denk et al., 1990). Among different multiphoton implementations (Zipfel et al., 2003; Zumbusch et al., 1999), second harmonic generation imaging (Roth and Freund 1980; Freund et al., 1986; Campagnola et al., 2001; Yeh et al., 2002; Campagnola and Lowe, 2003; Cox et al., 2003) is particularly suitable for investigating non-centrosymmetric structures. Second harmonic generation (SHG) is a nonlinear optical process that occurs only at the focal point of a laser beam (Shen, 1989). SHG is not much younger than laser, but the application of such a weak process to the imaging of cellular structures and functions is quite new and notable (Campagnola and Loew., 2003). Advancement in mode-locked laser (instead of a continuous wave, mode-locked laser emits short pulses in the range of ns to fs) makes SHG imaging of cells possible since one do not need such a high intensity which in fact cooks the cells right a way. Using chiral chromophores, chiral SHG imaging can be applied to otherwise impossible symmetric structures (Yan et al., 2006). Second harmonic imaging microscopy (SHIM) is based on nonlinear optical effect known as second harmonic generation. A laser scanning microscope using second harmonic generation as a probe is shown to produce high-resolution images of transparent biological specimens. SHIM has 
been established as a viable microscope imaging contrast mechanism for visualization of cell and tissue structure and function. A second harmonic microscope obtains contrasts from variations in a specimen's ability to generate second harmonic light from the incident light, whereas a conventional optical microscope obtains its contrast by detecting variations in optical density, path length, or refractive index of the specimen. SHG requires intense laser light passing through a material with a non-centrosymmetric molecular structure. Second harmonic light emerging from an SHG material is exactly half the wavelength (frequency doubled) of the light entering the material. The two-photon-excited fluorescence (TPEF) is also a two photon process. The TPEF loses some energy during the relaxation of the excited state, while SHG is energy conserving.

In the two photon absorption (Figure 1a) a fluorophore is simultaneously excited from the ground state through a virtual state to either the first or second excited state. In case if the fluorophore is excited to the second excited state a rapid nonradiative decay to the first excited state generally results in the same emission independent of excitation mode. The excitation of the fluorescent molecules occurs because of the nonlinear response of the molecules to the electric filed. It is common to assume that the effective excitation profile is simply given by the square of the (Gaussian-Lorentzian) laser beam focused by the objective. Two-photo excitation fluorescence is a four level resonant process involving effectively simultaneous absorption of two low energy photons via a virtual state to cause electronic excitation to a real excited molecular vibronic state followed by fluorescent emission. This emission will have an angular distribution determined by the molecular configuration and a fluorescence decay time determined by the electronic upper state lifetime (Figure 1a). The emitted photon has a wavelength slightly greater than the half wavelength of the excited source. For centrosymmetric or randomly-oriented fluorophores, the emission is isotropic. The fluorescence decay time is in the order of picoseconds or longer. Hence, fluorescence is incoherent.

Let us start the discussions with an overview of the nonlinear optical process from the point of view of physics, in general the nonlinear polarization can be written as;

$$
P=\chi^{(1)} E^{1}+\chi^{(2)} E^{2}+\chi^{(3)} E^{3}+\cdots
$$

where $P$ is the induced polarization. $\chi^{(n)}$ is the $\mathrm{n}^{\text {th }}$ order nonlinear susceptibility, and $E$ is the electric field vector. The first term describes the normal absorption and reflection of the light, the second term is the second harmonic generation (SHG) and the third one is third harmonic generation (THG). The SHG is of primarily electronic origin and is a non-resonant process. In the SHG two photons of the same wavelength coalesce to a virtual state within the specimen to form a single photon of energy exactly twice that of the incident photons (Figure 1b). The SH photon is generated almost instantaneously (within a few fs) so that the $\mathrm{SH}$ signal is coherent.

It is well known that the SHG does not arise from an absorptive process; the intense laser field induces a nonlinear polarization in a molecule(s) resulting in the production of a coherent wave which is twice times the incident frequency. The magnitude of the SHG wave can be enhanced when the energy of the SH signal overlaps with an electronic absorption band (Heinz et al., 1982). Let us go back to eq. 1, we will find that the SHG can result from both non-centrosymmetric environment and electric quadrupole interaction from samples with a large change in optical dielectric constant between the interfacial regions. This interaction will give rise to the SHG signal from centrosymmetric regions, but this 


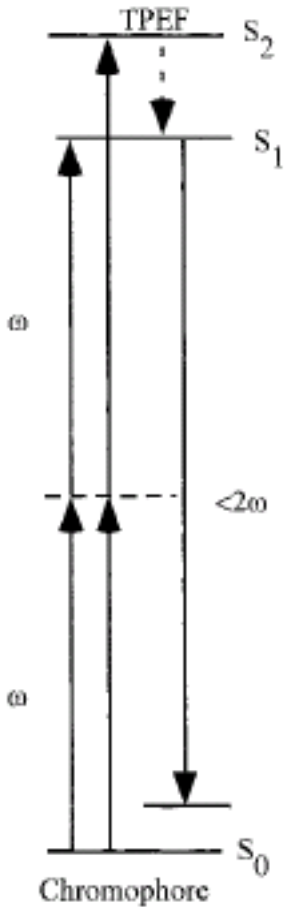

(a)

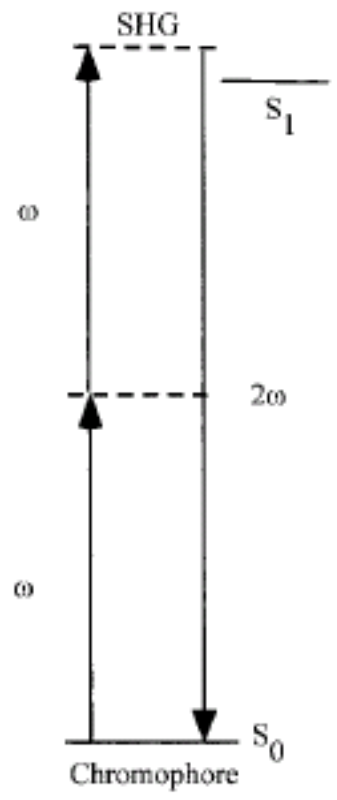

(b)

Fig. 1. (a) The photo-physical pathways for two-photon excitation fluorescence. (b) Resonance enhanced SHG.

contribution is very weak in comparison with the interfacial components. So the SHG will form the basis of a high resolution nonlinear optical imaging that possesses all of the benefits of multiphoton excited fluorescence microscopy. Resulting in reduction of the photo-bleaching and photo-toxicity, moreover using mode-locked laser infrared wavelength also will reduces the photo-bleaching and photo-toxicity.

The multi-functional technique enables us to see the true architecture of the specimen which doesn't produce auto-fluorescence. In order to acquire fluorescence imaging for these samples one needs to stain those with fluorescence dyes in order to acquire images representing the true architecture of the specimen. Sometimes the staining harm the living tissue (some of these dyes are toxic) so for such sensitive tissues one prefers to use the $\mathrm{SH}$ imaging. Moreover, it is well known that during an investigation of new samples and acquiring fluorescence images, looking in very details at the true architecture of the specimen is required to exposure it to the laser beam more than the well-known sample. The sample will undergo photo-bleaching and photo-damage, because there is a certain limit for the laser power and the time of exposure the sample to the laser beam. While the $\mathrm{SH}$ imaging process does not undergo photo-bleaching and photo-damage because of being a coherent process, which occurs within a few femtoseconds, and is known to leave no energy deposition to the interacted matters due to their virtual energy conservation characteristic, it 
is an advantage of the SH imaging when one needs to work with sensitive samples. Thereby one can investigate the true architecture of the sensitive samples and the new samples. Then one can move only the filter cube (Beamspliter cubes, see Fig. 2a) in order to acquire fluorescence images after well investigation of the true architecture of the sample. The other advantage of SHG is the ability of imaging highly ordered structural proteins without any exogenous labels (Campagnola et al. 2001). The SHG is an intrinsic process, imaging of intrinsic compounds avoids the complications of slicing and labeling, and samples can be investigated under physiological conditions.

Advances in the developments of second harmonic generation microscopy have provided researchers with novel means by which non-invasive visualization of non-biological and biological specimens can be achieved with high penetration and high spatial resolution. The emitted SHG photon energy is the same as the total absorbed excitation photon energy which provides the optical non-invasive nature desirable for microscopy applications (Gao et al. 2006). The inhomogeneity inherent to most biological specimen, and in particular, the internal structure of various cells, leads to generate high quality SHG images without any preconditioning such as labeling or staining that might induce undesirable effects in the living cell (Reshak, 2009). Historically, resolution in fluorescence optical microscopy has been limited by the Rayleigh criterion. The Rayleigh criterion states that two images are just resolved when the principal maximum (of the Fraunhofer diffraction pattern) coincides with the first minimum of the other (Born and Wolf, 1980). Techniques with better resolution than the Rayleigh criterion have recently been established, among which is harmonic excitation light microscopy (Frohn et al., 2000).

Second harmonic generation microscopy is a newly emerging microscopic method. Both backward and forward generated second harmonic imaging can reveal non-centrosymmetric and inhomogeneous structure of the object. In SHG microscopy, second harmonic light is generated at the focal point of a tightly focused short pulse laser beam. If the medium at the focal point is centro-symmetric and homogenous, the second harmonic waves generated before and after the focal point interfere destructively, resulting in zero net SHG (Boyd, 1992). However, when there are inhomogeneities near the focal point, such as an interface between two media, or if the material is non-centrosymmetrical, the symmetry along the optical axis breaks and a measurable amount of second harmonic is generated (Reshak et al, 2007; Lukins et al., 2003)). Due to its nonlinear nature, the second harmonic light is generated only in close proximity to the focal point (Yelin and Silberberg, 1999). This, in turn, leads to high lateral resolution and inherent optical sectioning of the sample facilitating the construction of three dimensional images (Mason, 1999; Helmchen and Denk, 2005).

The use of infrared light to excite fluorophores in light-scattering tissue has added benefits (Helmchen and Denk, 2005). Longer wavelengths are scattered to a lesser degree than shorter ones, which helps to increase penetration depth and image quality. In addition, the non-linear nature of the process is less likely to cause damage outside of the focal volume. In laser scanning two photons excited fluorescence microscopy, molecular excitation of fluorophores is achieved by the simultaneous absorption of two photons (within $\sim 5 \mathrm{fs}$ ) (Helmchen and Denk, 2005), which also provide intrinsic optical sectioning. The excitation of fluorophores usually requiring single-photon absorption in the ultraviolet is now achieved with a stream of strongly focused subpicosecond pulses of red laser light. The fluorescence emission increases quadratically with the excitation intensity so that fluorescence and photo-bleaching are confined to the vicinity of the focal plane as expected for cooperative two-photon excitation (Mason, 1999). This technique also provides 
unprecedented capabilities for three-dimensional, spatially resolved photochemistry, particularly photolytic release of caged effector molecules.

Similar to TPEF, the SHG is produced in only a small focal volume, permitting high resolution 3D optical sectioning of thick tissues. In contrast to TPEF, the SHG is an intrinsic and a coherent process. Coherent constructive or destructive interference of SHG provides extra hints to the ultrastructure of the sample details and their organization. SHG is very efficiently generated in chloroplasts (Chu et al., 2001). Chloroplasts in celery showed a signal in the SHG image which did not co-localize with the autofluorescence of the chlorophyll. Crystalline starch in starch grains is typically organized with the crystallites in a radial fashion, yielding a characteristic cross image in polarized light (Clowes and Juniper, 1962). This in turn means that SHG image will be orientation dependent (Cox et al., 2004). The significant SHG seen in biological materials arises from low local symmetry and the large nonlinear coefficient typical for biological molecules and structures (Lukins et al., 2003; Mason, 1999; Helmchen and Denk, 2005).

The SHG signal unlike fluorescence is highly asymmetric due to the phase matching condition (Cheng et al., 2002; Moreaux et al., 2000; Williams et al., 2005; Mertz and Moreaux, 2001). The forward and backward SH signals depend on the axial size of the object. If the axial size of the object is of the order of the $\mathrm{SH}$ wavelength or greater then the object exhibits forward directed SHG, whereas if the object axial size is less than one tenth of the wavelength, it is estimated to produce nearly equal backward and forward signals (Moreaux et al., 2000; Williams et al., 2005; Mertz and Moreaux, 2001; Reshak, 2009).

SHG strongly depends on the state of the polarization of the laser light and the orientation of the dipole moment in the molecules that interact with the light. It is therefore advantageous to control the laser's state of polarization, to maximize SHG (Lukins et al., 2003; Reshak, 2009).

Although the SHG in biological tissue was first demonstrated two decades ago (Freund et al., 1986; Fine and Hansen 1971; Roth and Freund 1980), SHG has only recently been used for biological imaging applications (Georgiou et al., 2000; Campagnola et al., 1999; Gauderon et al., 1998; Moreaux et al., 2000) because the SHG and TPEF involve different contrast mechanisms, they can be used in tandem to provide complementary information regarding tissue structure and function (Zoumi et al., 2002). The SHG signals depend on the orientation, polarization, and local symmetry properties of chiral molecules, whereas the TPEF results from the nonlinear excitation of molecules fluorescence (Zoumi et al., 2002).

Researchers have been interested in optical methods for driving micro machines for a few years, with options often focusing on light-induced rotation of absorbing microscopic particles using elliptically polarized laser beams or beams with helical phase structures. These experiments, however, involved trapping absorbing particles and thus limited power to avoid heating effects. This power restriction, in turn, limited rotation rates to a few hertz. Chloroplasts are the photosynthetic organelles of plant cells. They are commonly believed, and virtually all textbooks show them, to be ellipsoids of revolution flattened along the axis (Rezende-Pinto, 1972), or shaped like a dinner plate, flattened with curried edge (Sarafis, 1998). Most of the studies on their structure and shape have been made with light and electron microscopy (Wildman et al., 1980). Such studies, however, are largely limited to observation of two dimensional cross-sections because of the limited depth of field of the optical microscope and the need to use thin sections in electron microscopy. While studies by light and electron microscopy have been extensive, the preparation methods for these investigations involve treatment with fixatives and staining agents. Such treatment may alter the shape and the gross morphology of the organelles. Furthermore, a problem with 
assessing chloroplast shape is that the angle of presentation is usually a matter of chance. Tactile maneuvering of chloroplasts has not widely attempted. To overcome these problems the Multi-functional Two-Photon Laser Scanning Microscopy is used. The MF-2PLSM recently established (Reshak, 2009) by combing three platforms of laser scanning microscopy; the fluorescence microscopy, harmonic generation microscopy and polarizing microscopy. With MF-2PLSM one not need to stain the sample by utilizing the second harmonic imaging (SHI). Utilizing the non-invasive nature of the second harmonic imaging to record the rotation of the chloroplast with increasing/ decreasing the laser's power and follow the movements and the orientation of the chloroplast while illuminated by linearly polarized laser and increase the laser's power to see the react of the chloroplasts. The reaction of the chloroplasts comes as movement and rotation, trying to protect the photosynthetic system from being damaged while it is exposures to high intensity laser. The chloroplasts reorient themselves to make the orientation of the dipole moment in the molecules perpendicular to the laser's polarization direction. This rotation leads to very less amount of light will penetrate inside the chloroplasts. Then we try to increase the laser's power to monitor the behaviors of the chloroplast and as a consequence the chloroplasts move and rotate faster, which makes it possible to manufacture micromotors for micromechanical systems. The goal is to extend the concept of the optical torque on in vivo birefringent biological particle to a new optical method for driving micromachines.

\section{Laser sources and imaging system}

The experimental arrangement is shown in Fig. 2. We used an inverted i-mic 2 microscope (Till-Photonics, Grafelfing, Germany), (http://www.till-photonics.com/Products/imic.php) equipped with Ti:sapphire femtosecond laser with a tuning range of $690 \mathrm{~nm}$ to $820 \mathrm{~nm}$. The laser is a Tsunami 3941-M3B pumped by a Millennia-V, 5W solid-state pump laser (SpectraPhysics). This laser was used to generate linearly polarized pulses at $810 \mathrm{~nm}, 20 \mathrm{~mW}$, and 80 fs pulse width for fluorescence excitation and SHG. A beam expander was used to fill the back aperture of the objective. The excitation light was directed onto a pair of galvanometer XY scanners (Yanus-Till-Photonics) (http://www.till-photonics.com/Products/imic.php). The scanned excitation light was focused onto the specimen through the microscope objective (Olympus uplanApo/IR 60×/1.20 water immersion) in order to scan the specimen in the $x-y$ direction at the focal plane. The stage of the microscope is driven by a computer controlled motor to take the specimen to different $z$ positions following each $x-y$ scan. The scanning mirrors are metal coated (silver) with a good thermal resistance (Diaspro, 2001). Further components from the setup in Fig. 2 are the dichroic mirrors (mirror 1: Omega 475DCLP for SHG (Fig. 3a), interference blue emission filter 1 (Fig. 3b), mirror 2: Q565LP for TPEF (Fig. 3c), emission filter 2: red glass 665 nm, PMTs: Hamamatsu R6357, objective 1: Olympus uplanApo/IR 60×/1.20 water immersion, objective 2: Zeiss 40×/1.2W korr).

The collecting efficiency of SHG signals is highly dependent on the numerical aperture (N.A.) of the objective (Cox et al., 2004; Han et al., 2005; Pantic-Tanner and Eden, 1999; Faludi-Daniel et al., 1978; Sarafis, 1998). Additional infrared beam block filters (HQ700SP-2p 58398) were placed in front of each PMT to ensure that illumination light was effectively suppressed and only TPEF and SHG signals were recorded. For SHG imaging, optical filtering is achieved with an interference filter centered on the expected SHG frequency. The configurations of the two PMTs were identical for both TPEF and SHG imaging. This setup 


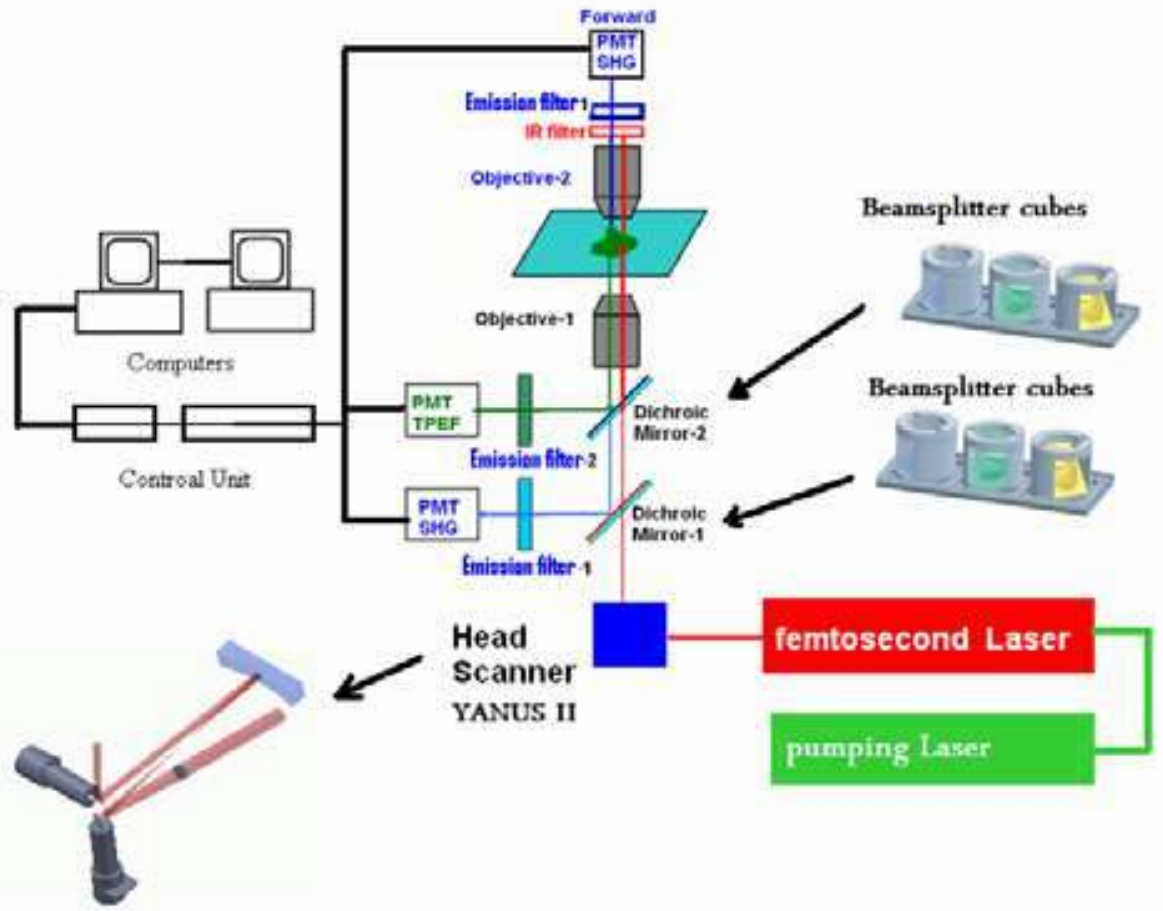

(a)

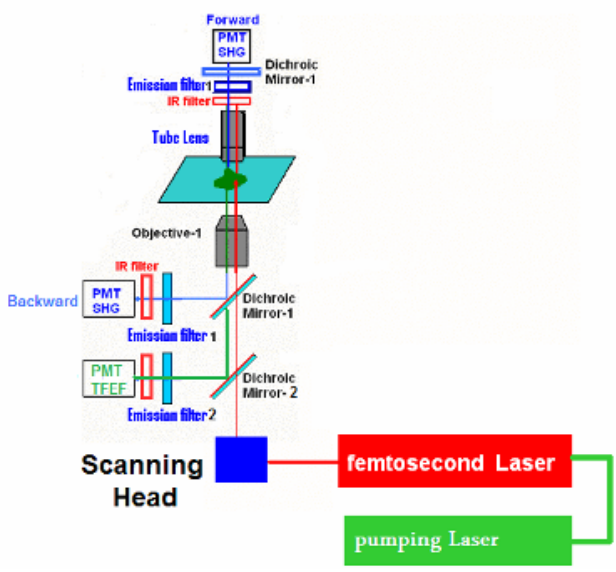

(b)

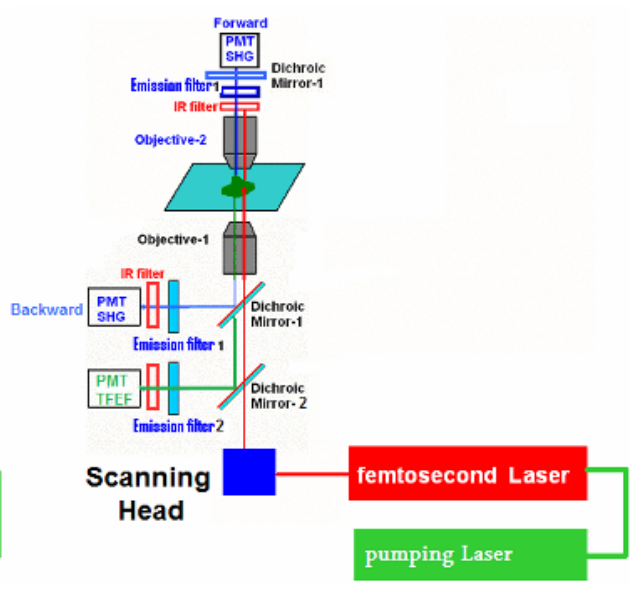

(c)

Fig. 2. (a) The experimental setup using two objectives for collecting the forward and the backward SHG signals. (b) Tube lens for collecting forward SH signals and objective for collecting the backward SH signals. (c). Objective for collecting forward SH signals and another objective for collecting the backward SH signals. 


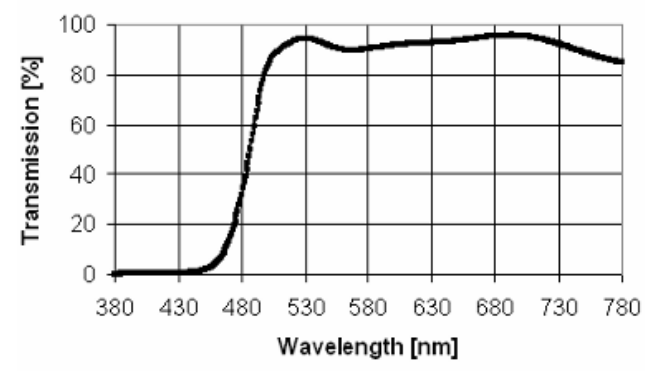

(a)

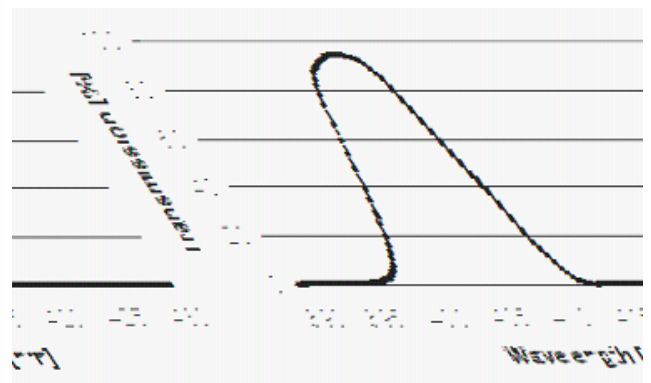

(b)

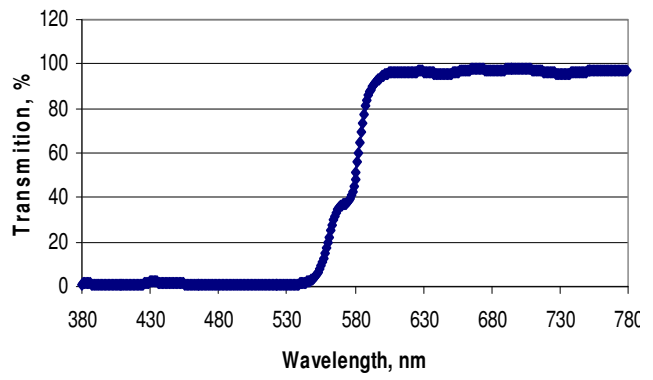

(c)

Fig. 3. The characterizations of the filters used for collecting the SHG and fluorescence; (a) Dichroic mirror Omega 475CLP for SHG. (b) Emission filter 1, for SHG. (c) Dichroic mirror Q565LP.

will enable the simultaneous measurement of SHG (Han et al., 2005) in the forward and backward directions. The signals from the photomultipliers are reconstructed by a computer into images. Images were obtained in stacks stepping along the $\mathrm{z}$-axis with $0.5 \mu \mathrm{m}$ steps. The microscopy is controlled via a standard high-end Pentium 4 PC and linked to the electronic control system via an ultrafast interface.

For separating the $\mathrm{SH}$ signals in the forward and backward directions and to suppress the fluorescence the dichroic mirrors Omega 475DCLP and interference blue emission filter centered on the expected SHG frequency were used. To ensure that only the SH signals were recorded and effectively suppressed any other signals an additional infrared beam block filters (HQ700SP-2p 58398) were placed in front of each PMT. In order to enhance the forward SH signals different objectives with different numerical aperture and immersion medium were used: Tube lens ( $\mathrm{f}=16 \mathrm{~mm}, \mathrm{~d}=12 \mathrm{~mm}$, PCX Lens fromEdmund), (http://www.edmundoptics.com/onlinecatalog/displayproduct.cfm?productID=2595), Olympus uplanFLN $10 \times / 0.3$, Zeiss $40 \times / 1.2$ korr, water immersion, and Olympus uplanApo/IR 60×/1.20 water immersion. 


\section{Material}

In this work the moss Plagiomnium affine plant was used (see Fig. 4). This moss plant has a leaf of single cell layered. A leaf was detached from plants of the moss Plagiomnium affine by a watchmakers forceps from a darkened part of the moss plant. The leaf was mounted between two coverslips in water and the edges of the coverslip of smaller dimensions was sealed to the lower larger coverslip by means of nail varnish and allowing it to dry. The paired coverslips were placed on the stage of a Till-Photonics microscope and illuminated with a Titanium sapphire laser at $810 \mathrm{~nm}$ (linearly polarized laser). The objectives were aligned relative to one another and focused on the leaf. A set of images was captured over a time interval of half an hour where the chloroplasts changed their SHG due to their reorientation.

Because of some uncertainty in the chloroplast imaging as shown by Chu et al. 2001, who looked at chloroplasts containing starch grains which are strong sources of second harmonic light we used a moss Plagiomnium affine with large chloroplasts devoid of starch by keeping the plants in the dark for approximately three weeks. The typical size of these chloroplasts vary between 5-10 $\mu \mathrm{m}$. Short pulses should be used and average laser power should be kept low to prevent heating of the sample as well as unwanted one-photon absorption and to reduce the risk of highly nonlinear photodamage (Denk et al 1995). $\lambda / 2$ plate was used to control or maximize the status of the laser's polarization at the sample.

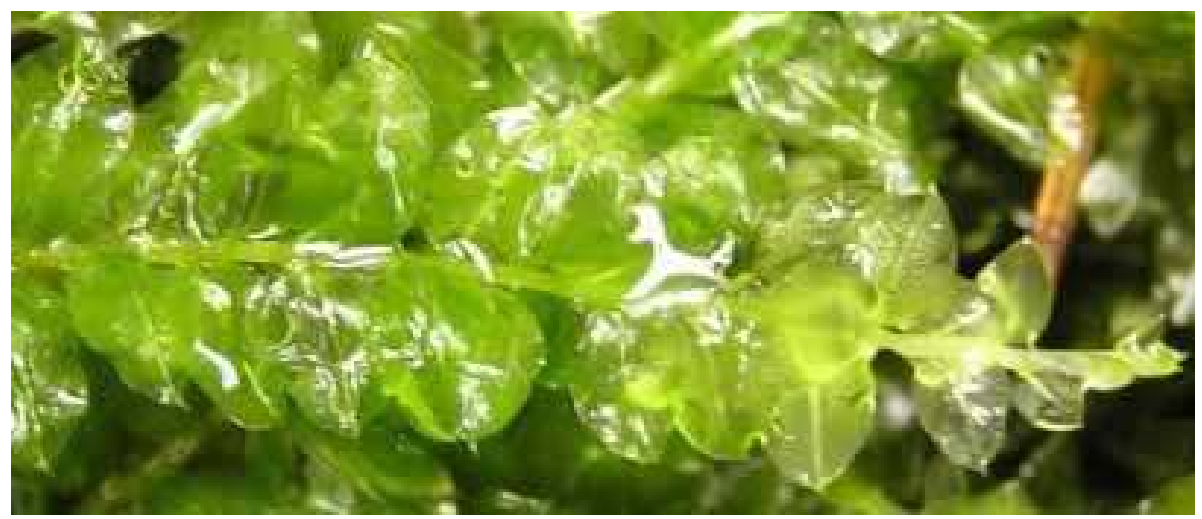

Fig. 4. The moss Plagiomnium affine plant.

\section{Results}

Fig. 5, show time-series images using the experimental setup shown in Fig. 2. The backward SHG signal was collected using the objective Olympus uplanApo/IR 60×/1.2 water immersion. In this figure we labeled some chloroplasts as a reference for the discussion of the rotation and the movements of the chloroplast. The $\mathrm{SH}$ signal showed no signs of bleaching during acquisition of repeated images from a given area showing no damage to the structure. This is to be expected since SHG is a coherent process, unlike fluorescence, and no energy is lost (Mark et al., 2003). There is a signal from membrane-filled regions devoid of starch grains. The SHG intensity altered dramatically with time as seen in the time-series of images shown in Fig. 5 which is analyzed in Fig. 6. 


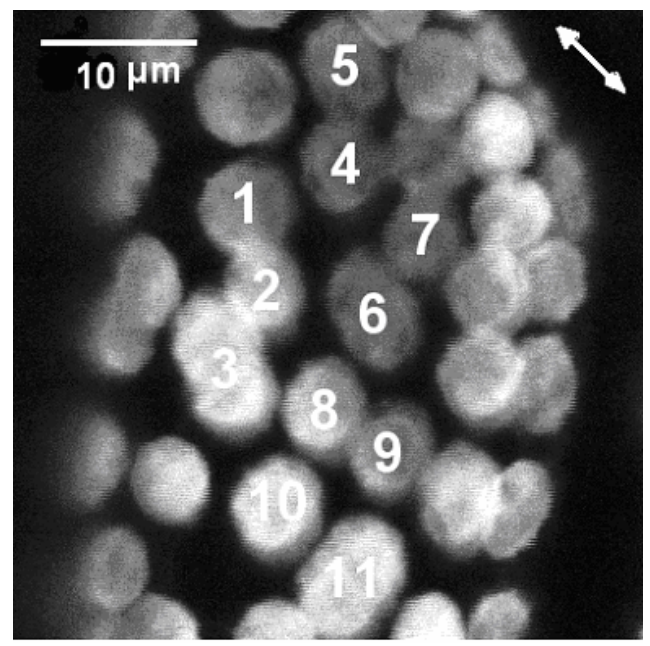

Fig.5a-Backward SHG 60x 1.2w

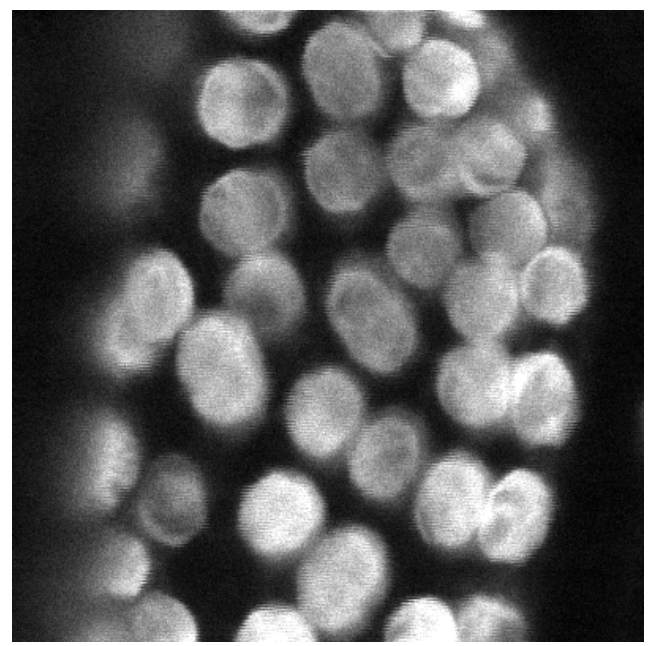

Fig.5c-Backward SHG 60x 1.2w

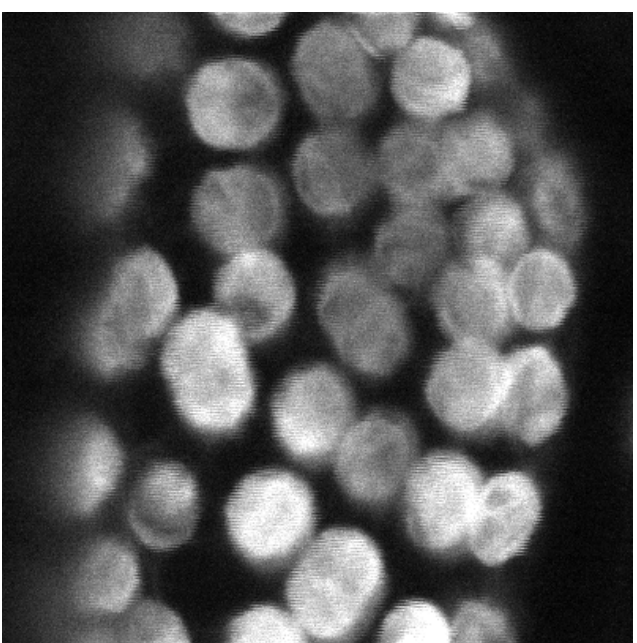

Fig5b-Backward SHG 60x 1.2w

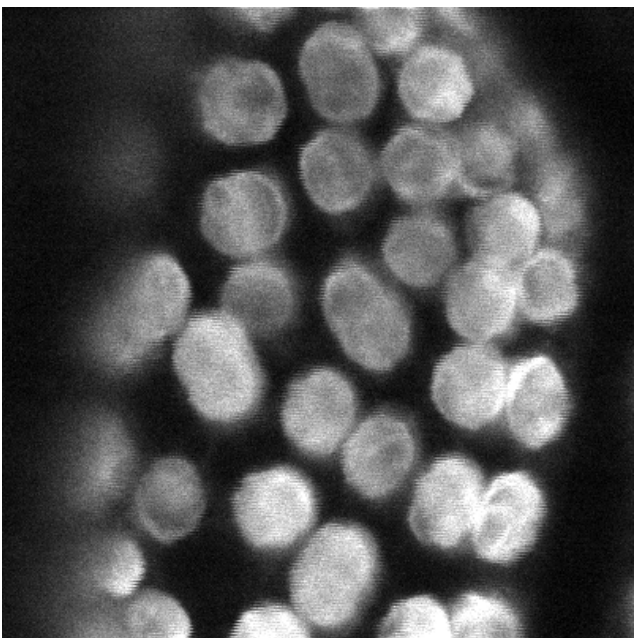

Fig.5d-Backward SHG 60x 1.2w 


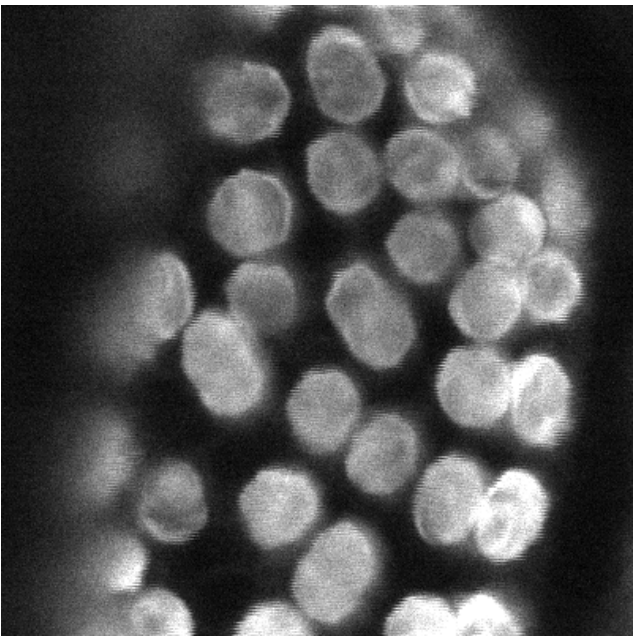

Fig. 5e. Backward SHG 60x 1.2w

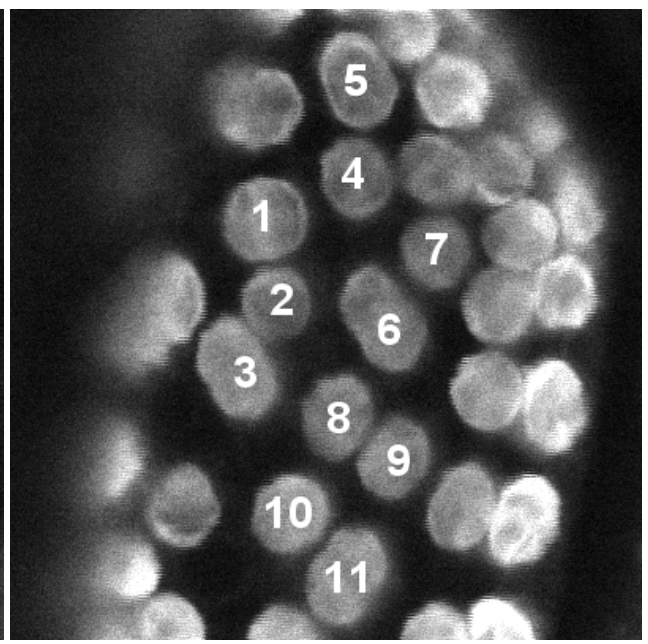

Fig. 5f. Backward SHG 60x 1.2w

Fig. 5. Time-series SHG images of Plagiomnium affine leaf showing the movement and the rotation of the chloroplast $(\mathrm{a}, \mathrm{b}, \mathrm{c}, \mathrm{d}, \mathrm{e}, \mathrm{f})$ Backward SHG signal using the objective Olympus uplanApo/IR60x/1.2 water immersion. The double arrow shows the direction of polarization of the $810 \mathrm{~nm}$ laser beam. Some chloroplast labeled by numbers $1,2,3, \ldots$ as a reference for the discussion the rotation and the movements of the chloroplast in the timeseries images.

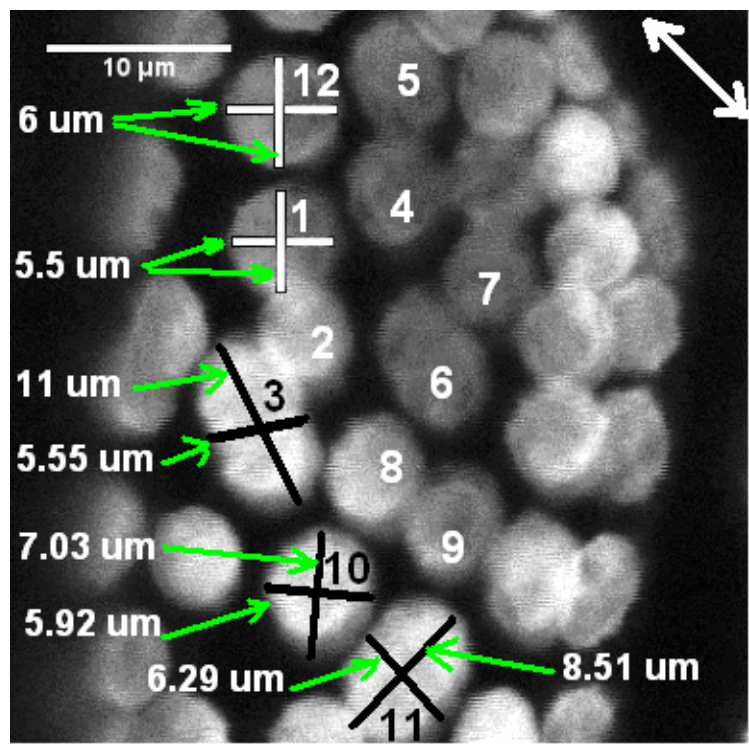

Fig. 5aa. (the first image of the time series SHG images-Fig.5a) shows the size of some selected chloroplast in the first stage of being illuminated by linearly polarized laser. 


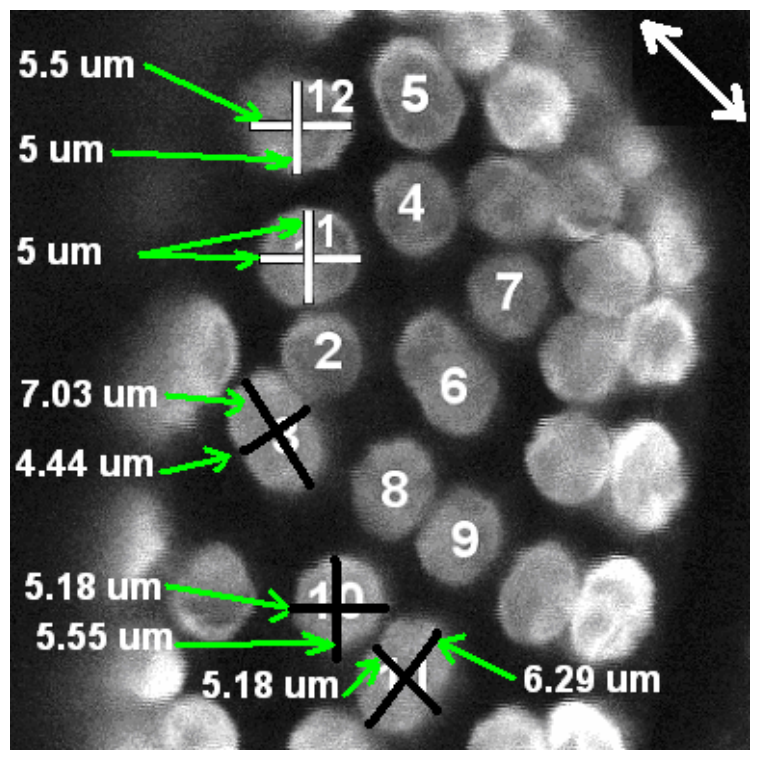

Fig. 5ff. (the last image of time series SHG images-Fig.5f) Shows the size of some selected chloroplasts in the final stage when it is illuminated by linearly polarized laser. It is clear that the sizes of these chloroplasts have changed due to the rotation of the chloroplasts when they were being illuminated by linearly polarized laser.

Fig. 6 shows the time-dependence of the average backward SHG signal in figure 5 of the inboxes each containing a chloroplast (see corresponding numbers in Fig. 5a). Using successive cross-correlations image drift was compensated for. We notice significant changes in the SHG intensity over time. The chloroplasts rotated resulting in changing the axial aspect of the chloroplast (the changing in the intensity signal). This rotation causes a reduction/ increase in the brightness (intensity) of the signals depending on the aspect presented to the laser.

In Figs. 5aa and 5ff, we measured the size of some selected chloroplasts at the first stage of being illuminated by linearly polarized laser and at the final stage when they rotate. It is clear that the apparent sizes of these chloroplasts have been changed due to their rotation when they illuminated by linearly polarized laser. Chloroplasts rotate as a result of the cells which contain them being illuminated by blue light (Haupt and Scheuerlein, 1990).

As axial thickness increased the SHG signal will decrease in intensity. The SHG stems from chloroplasts devoid of starch, thus clearly demonstrating the generation of SH from the noncentrosymmetric membranes opposed to each other in the grana. It is known that chloroplasts exhibit strong birefringence, with high local values, mostly originating from grana and inclued starch grains (Garab et al., 2005; Goedheer, 1955). The size and sign of the birefringence are such that the resulting anisotropic interaction with a linearly polarized laser beam significantly contributes to the torque orienting the chloroplast. Optical reorientation by linearly polarized light occurs when $n_{e}>n_{0}$, and thus optical field tends to orient the director parallel to its electric vector $\left(n_{e}\right.$ and $n_{0}$ denote the extraordinary and ordinary refractive indices, respectively). Taking into account their asymmetric shape 


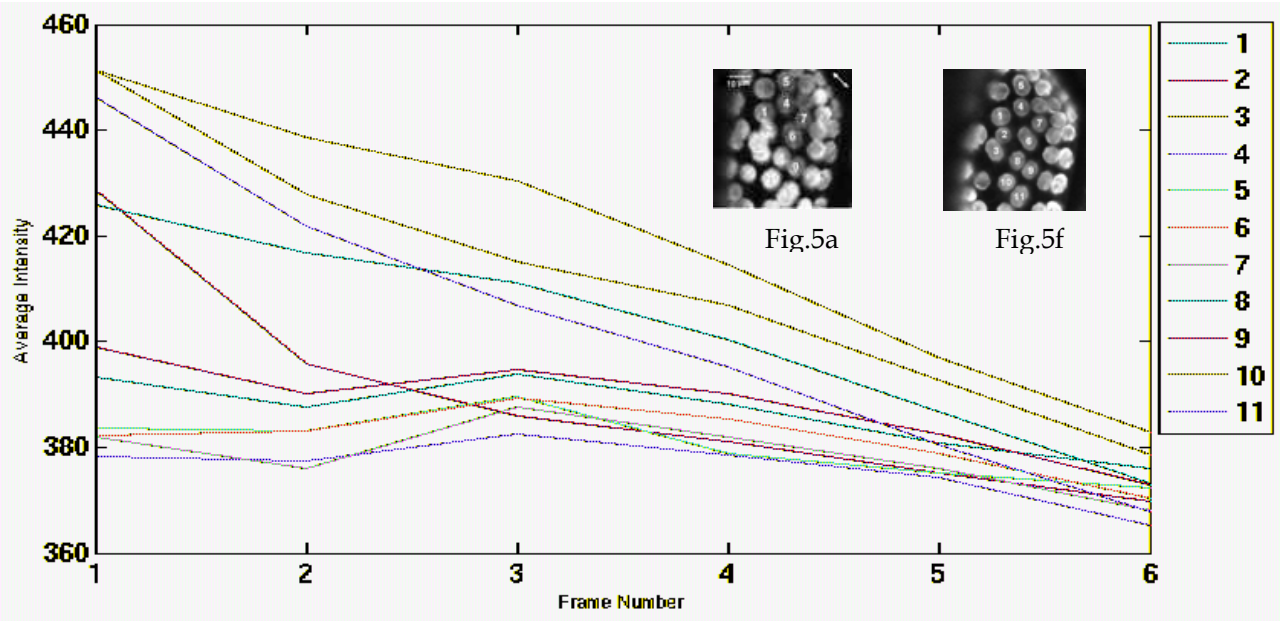

Fig. 6. Time dependence (corresponding to the backward generated SH shown in figure 5af) of the chloroplast-averaged backward SHG signal as numbered in Fig. 5a.

and the birefringence, biological entities may align in accordance with a preferential axis at the focus of the laser beam.

Figs.7 to 10 show the forward and the backward SHG structure of Plagiomnium affine leaf. The backward SHG signal was collected using the objective Olympus uplanApo/IR 60×/1.2 water immersion and the forward SHG signal was collected with a Zeiss $40 \times / 1.2$ water immersion objective, which show that the collecting efficiency of SHG signals is highly dependent on the numerical aperture (N.A.) of the objective (Cox et al., 2004; Han et al., 2005). In the right panel of each image the multicolor overlays of the respective forward (green) and backward (red) images were shown.

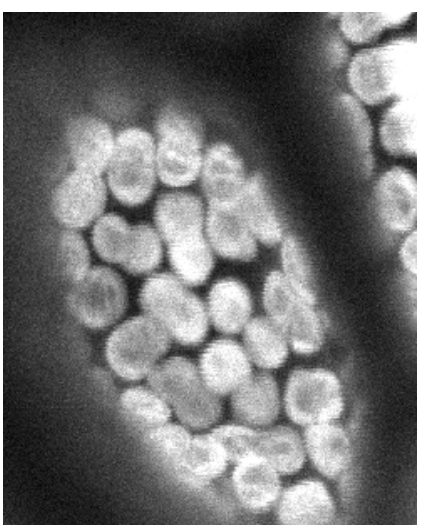

Fig. 7a.

Backward SHG 60x 1.2w

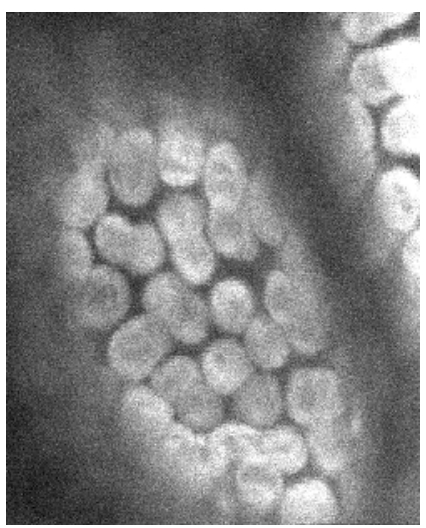

Fig. $7 b$.

Forward SHG 40x 1.2w

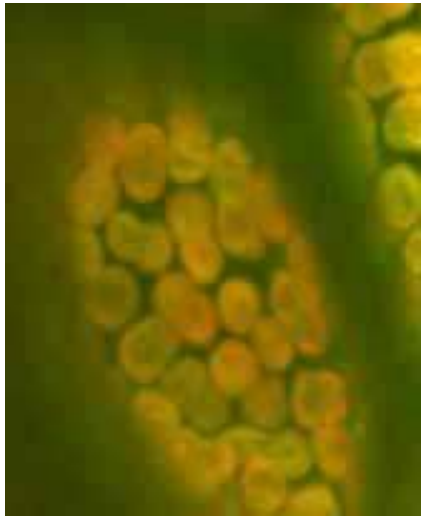

Fig. 7c.

Forward/Backward SHG 


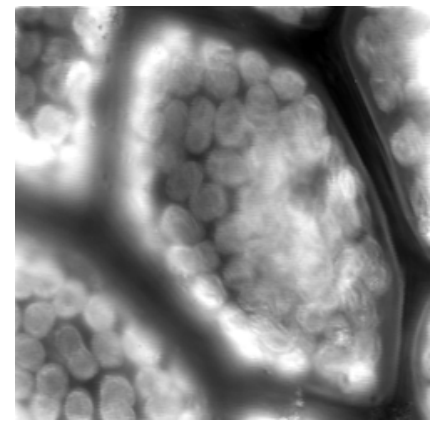

Fig. 8a.

Backward SHG 60x 1.2w

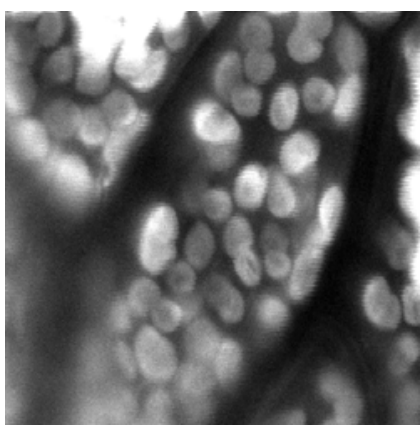

Fig. 9a.

Backward SHG 60x 1.2w

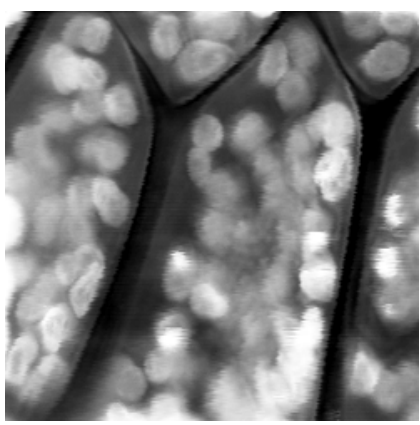

Fig. 10a.

Backward SHG 60x 1.2w

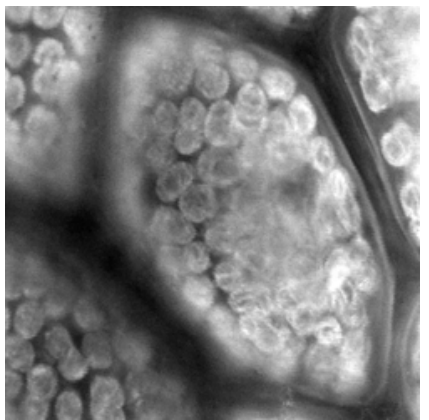

Fig. $8 b$.

Forward SHG 40x 1.2w

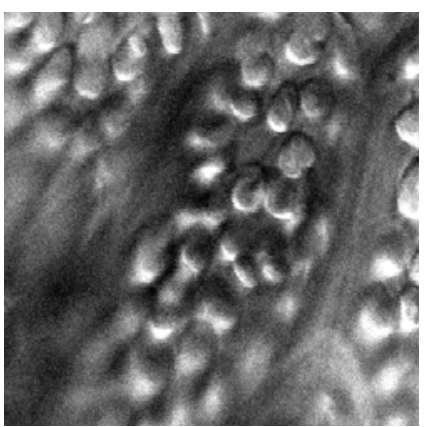

Fig. $9 \mathrm{~b}$.

Forward SHG 40x 1.2w

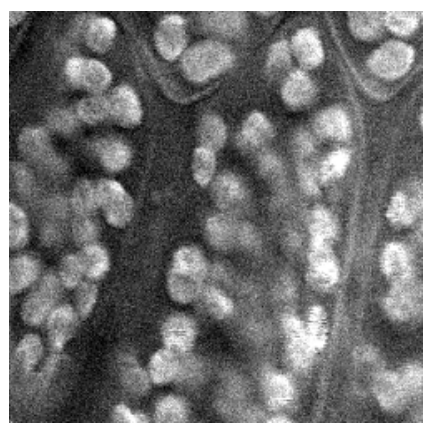

Fig. $10 b$.

Forward SHG 40x 1.2w

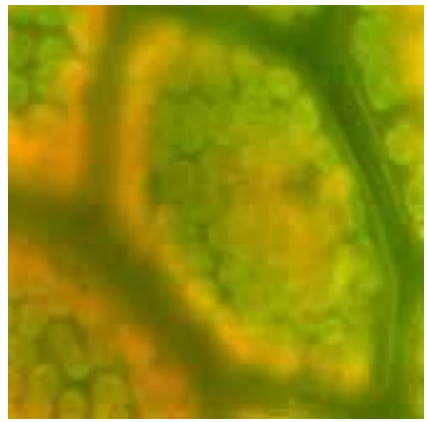

Fig. 8c.

Forward/Backward SHG

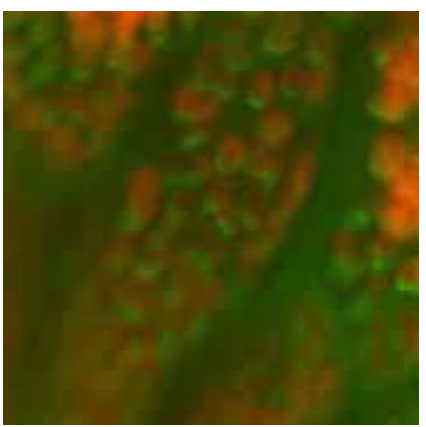

Fig. 9c.

Forward/Backward SHG

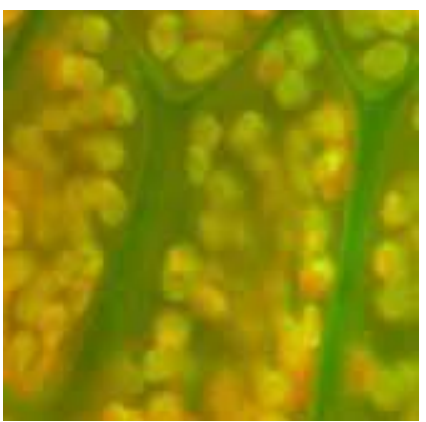

Fig. 10c.

Forward/Backward SHG

Figs. 7 to 10. shows SHG structure of Plagiomnium affine leaf. (a) Backward SHG signal using the objective Olympus uplanApo/IR 60×/1.2 water immersion. (b) Forward SHG signal was collected by a ZEIS $40 \times / 1.2$ water immersion objective. (c) Multicolor overlays of the respective forward (green) and backward (red) images. 
Fig. 11 shows the fluorescence which comes from the chloroplast, i.e the same area which produces the SHG signals. It is well know that the SHG is very efficiently generated in chloroplasts (Chu et al., 2001). Chloroplast in celery showed a signal in the SHG image which did not co-localize with the autofluorescence of the chlorophyll and the signals were mostly due to starch. We have used the dichroic mirror Q565LP (Fig. 3c) which reflects the wavelength from $540 \mathrm{~nm}$ and above. We have to mention that here we did not use an emission filter for this filter set, so the PMT collected all the fluorescence starting from 540 $\mathrm{nm}$ and above.

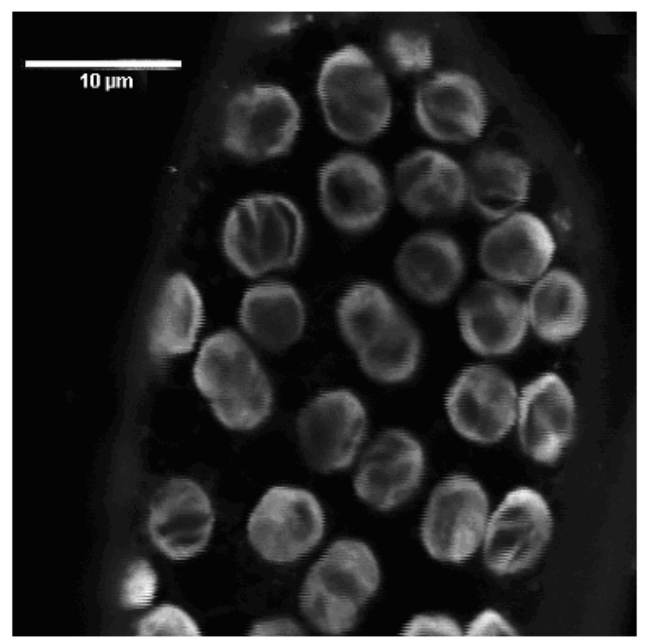

Fig. 11. Shows the fluorescence coming from the chloroplast, i.e the same area which produces the SHG signals.

\section{Discussion}

Chloroplasts in Plagiomnium affine leaves which were imaged came from a single cell layer lamina. The chloroplasts were disposed parallel to the upper and lower walls in all the cells of the lamina with the chloroplasts main axis oriented within the image plane. SHG signals were obtained in both directions with the forward signal predominating and these increased considerably when the chloroplasts rotated upon being illuminated by linearly polarized pulses of paired photons which are effectively equivalent to blue light at half the wavelength of the irradiating laser (Moreno et al., 2004). This chromatically equivalent blue range caused chloroplasts to rotate in a light causing orientation parallel to the side walls of the leaf cells. Starch grains are not involved thus eliminating a possible source of confusion. SHG indicates the non-centrosymmetric nature of the chloroplast membranes especially when aggregated into grana.

The chloroplast grana and intergranal membranes are distinguished by their birefringence (Pantic-Tanner and Eden, 1999). A low intrinsic birefringence is expected in intergranal membranes and a high birefringence where the membranes are stacked. The grana were expected to give a stronger $\mathrm{SH}$ signal than the intergranal regions with unstacked membranes. During the rotation of the chloroplast caused by linearly polarized high light intensity designed to present a smaller interface to the incoming light, the SHG increased 
considerably compared with the intergranal regions. Faludi-Daniel et al. 1978, refer to the structural distinctness of the chloroplast regions. Sarafis, 1998, reviewed and showed the range of chloroplast structure in higher plants in both deep shade plant leaves and dry resurrection plant leaves indicating a large variation in granal size which is expected to be shown by future second harmonic studies of these chloroplasts. We notice that the light absorption and the amount of fluorescence observed depend on the orientation of the chloroplast with respect to the polarization of the exciting photoactive light (Lukins et al., 2003).

\section{Conclusions}

We have demonstrated the simultaneously measured forward and backward SHG for the chloroplast of the Plagiomnium affine leaves. The SH signals are generated by a tightly focused short pulse laser beam. The SH signals are collected by two objectives to form forward and backward second harmonic digital images. These signals are dependent on the axial aspect of the chloroplast and that the back-scattered image was brighter than the forward scattered image. The image intensity also depended on the orientation of the chloroplast in relation to the illuminating polarization direction. A light-induced re-orientation in dependence of the illumination intensity could be observed. We found that the chloroplasts are moving and rotating when we expose the leaf to laser light for long time.

\section{Enhancing the resolution of the forward Second Harmonic imaging of the multi-functional two-photon laser scanning microscope}

Figs. 12a-e show the forward and backward SH imaging of Plagiomnium affine leaf using different objective and immersion imaging medium as shown in Figs. 2, 13 and 14. The backward SHG signal was collected using the objective Olympus uplanApo/IR 60×/1.2 water immersion and the forward SHG signal was collected by using different objectives; Tube lens( $\mathrm{f}=16 \mathrm{~mm}, \mathrm{~d}=12 \mathrm{~mm}$, PCX Lens from Edmund), Olympus uplanFLN $10 \times / 0.3$, Zeiss $40 \times / 1.2$ korr, water immersion, and Olympus uplanApo/IR 60×/1.20 water immersion. The resolution of the forward SHG signals increased dramatically with raising the numerical aperture when the objective is designed to operate with an immersion medium such as water between the front lens and the specimen coverslip, which shows that the collecting efficiency of SHG signals is highly dependent on the numerical aperture of the objective as seen in the series of images shown in Figs. 12a-e. The SHG stems from chloroplast devoid of starch, such clearly demonstrating the generation of SH from the noncentrosymmetric membrane apposed to each other in the grana.

From Figs. 12a-e, we note that by using low N.A. tube lens with air gap, to collect the forward SH signal we gain an image with gray background (see Fig. 12b). This gray background results from the interface glass-air, also the rays, that come from the specimen will bend away from the front lens of the upper objective due to the big difference in the refractive indices of the glass (1.5) and air (1.0) (see Figs. 13a, 14a, and 15). A step toward, in order to modify the forward $\mathrm{SH}$ images the tube lens was replaced with the objective Olympus uplanFLN 10×/0.3 (low N.A. with air gap), a significant increase in the intensity of the image was noticed (see Fig. 12c) indicating that more rays (diffraction orders) were collected by the Olympus uplanFLN $10 \times / 0.3$ objective. But the gray backgrounds are still there because of glass-air interface. Dramatic enhancement was noticed when the Olympus 
Second Harmonic Generation Signal from

uplanFLN 10×/0.3 objective was replaced by Zeiss $40 \times / 1.2 \mathrm{~W}$ korr objective (see Fig. 12d) indicating that by using high N.A. water immersion objective higher diffraction orders were collected (see Figs. 13b, 14b and 15). This enhancement attributed to the fact that the refractive index of the water (1.33) is closer to the refractive index of the glass (1.5) then the oblique rays will bend toward the front lens of the objective resulting to represent the true architecture of the specimen. In order to gain better quality images for the forward $\mathrm{SH}$ images the objective Zeiss $40 \times / 1$.2W korr was replaced by Olympus uplanApo/IR $60 \times / 1.20$ water immersion objective (see Figs. 12e, 14c and 15).

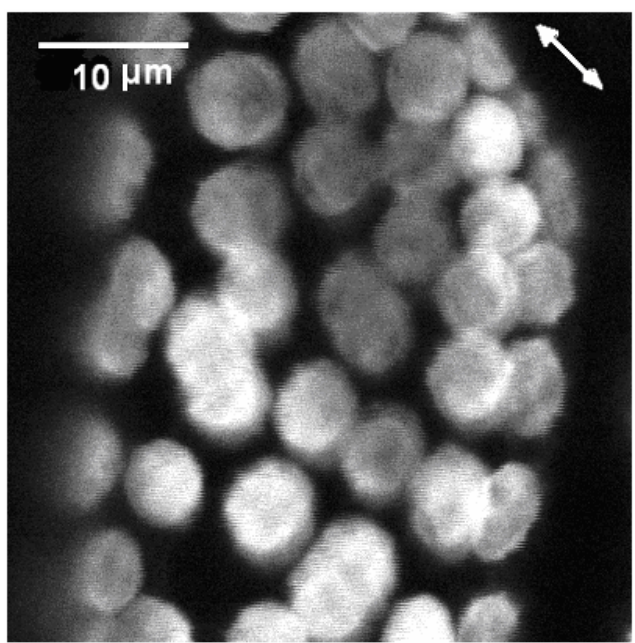

Fig. 12a.

Backward SHG with Olympus 60x 1.2w

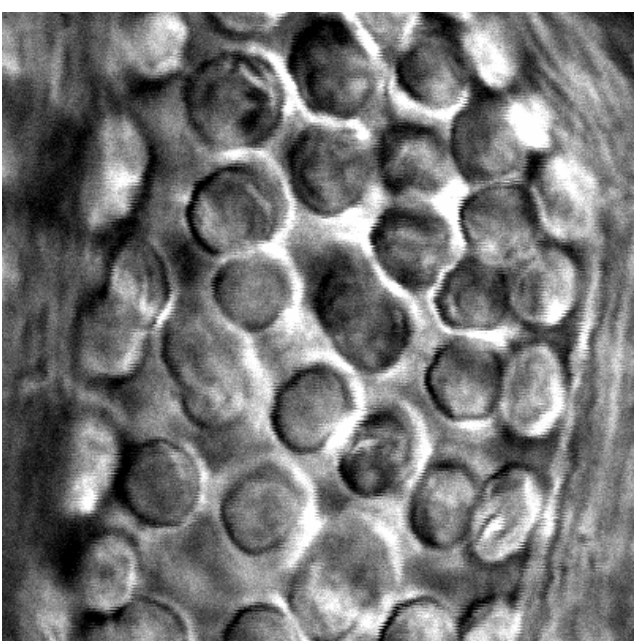

Fig. 12c.

Forward SHG with Olympus 10x

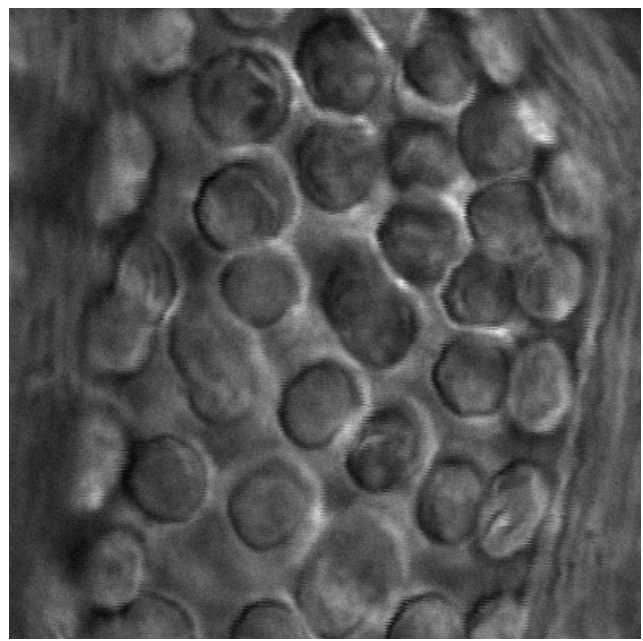

Fig. 12b.

Forward SHG with Tube lens

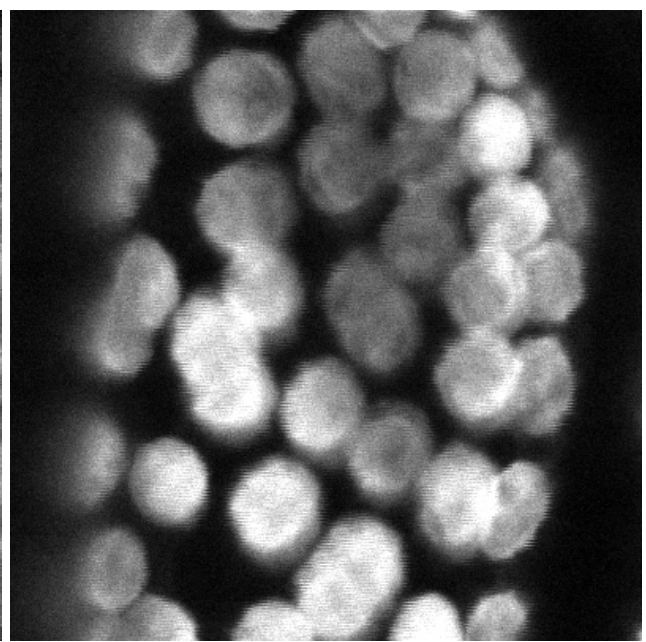

Fig. 12d.

Forward SHG with Zeiss40x 1.2w 


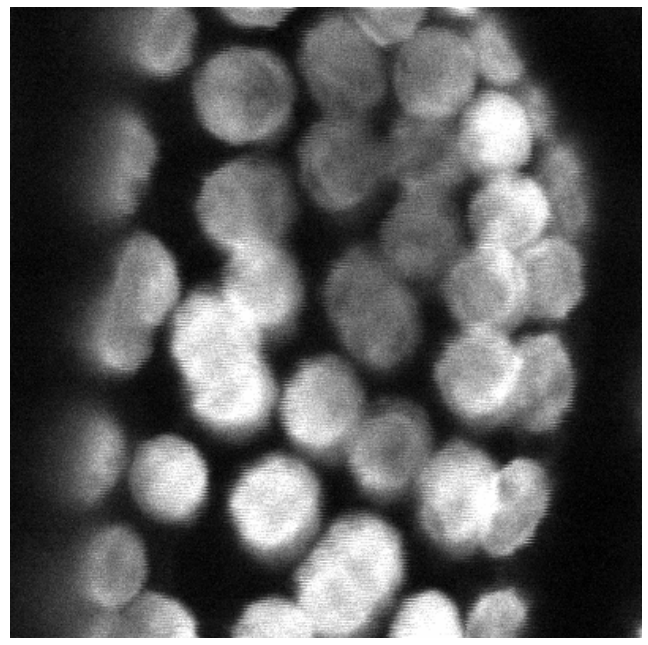

Fig. 12e. Forward SHG with Olympus 60x 1.2w

Fig. 12. SHG images of Plagiomnium affine leaf show the chloroplast (a) Backward SHG signal collected by using the objective Olympus uplanApo/IR 60×/1.2 water immersion. The double arrow shows the direction of polarization of the $810 \mathrm{~nm}$ laser beam. (b) Forward SHG signal using Tube lens. (c) Forward SHG signal using Olympus uplanFLN 10×/0.3 (d) Forward SHG signal using Zeiss $40 \times / 1$.2W korr objective (e). Forward SHG signal using the objective Olympus uplanApo/IR 60×/1.2 water immersion.

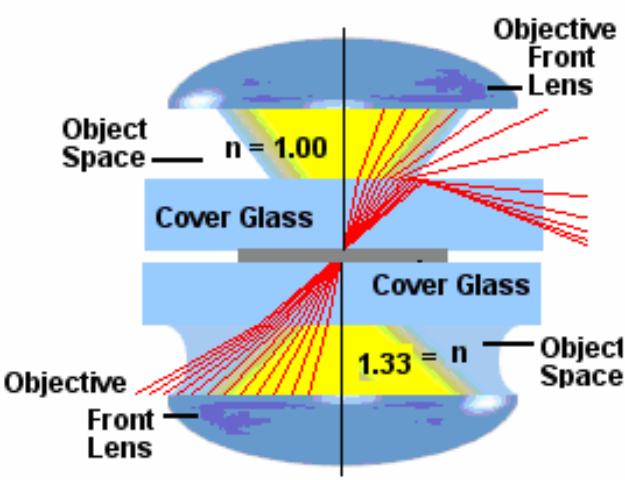

(a)

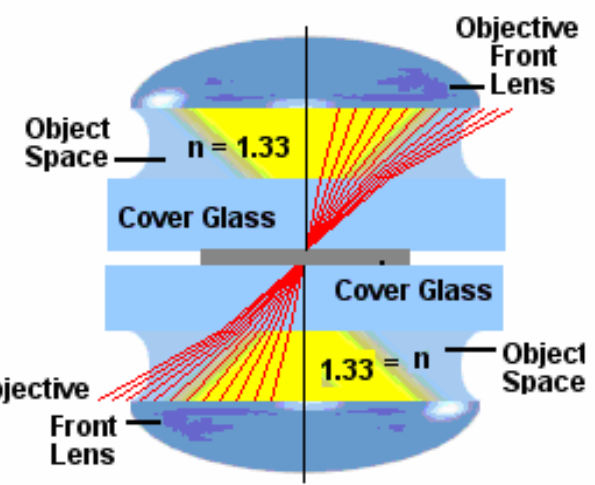

(b)

Fig. 13. The forward and backward objective with different numerical aperture and immersion medium: (a). air for the upper objective (which is used to collect the forward SH signals) and water for the lower objective (which is used to collect the backward SH signals). (b). water for both upper and lower objectives. 


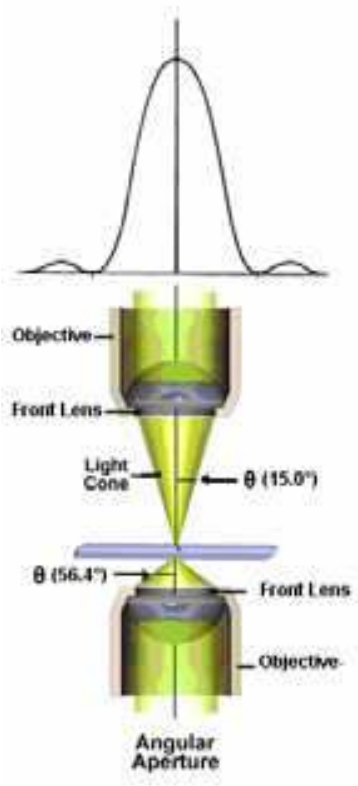

(a)

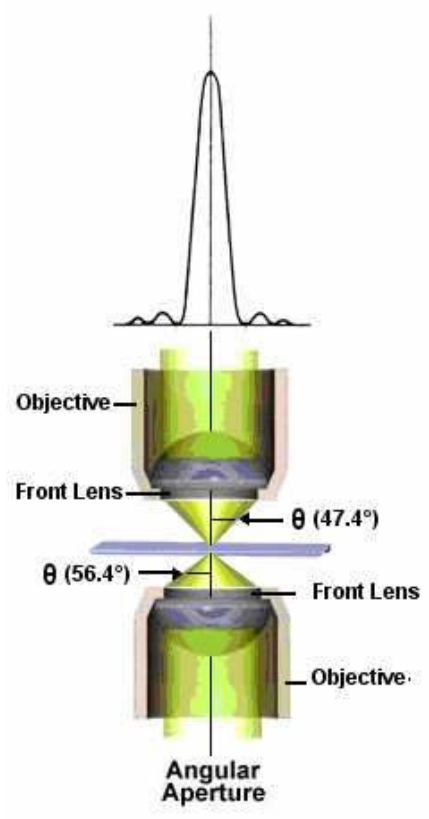

(b)

Forward with Zeiss $40 \times 1.2 \mathrm{w}$

Backward with Olympus $60 \times 1.2 \mathrm{w}$

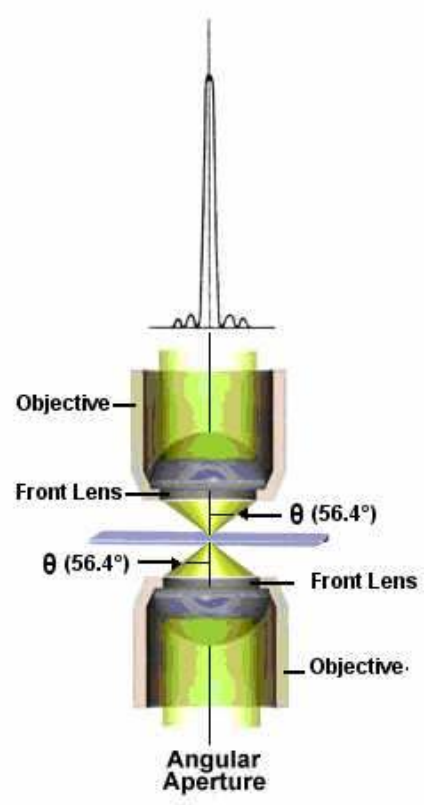

(c)

Forward with Olympus $60 \times 1.2 \mathrm{w}$

Backward with Olympus $60 \times 1.2 \mathrm{w}$

Fig. 14. The forward and backward objectives with different numerical aperture and immersion medium: (a). Olympus uplanFLN 10×/0.3 with air gap for the upper objective (which is used to collect the forward SH signals) along with the diffraction orders which can be collocated by the upper objective, and Olympus uplanApo/IR 60×/1.2 water immersion for the lower objective (which use to collect the backward SH signals). (b). Zeiss $40 \times / 1.2 \mathrm{~W}$ korr objective for the upper objective along with the diffraction orders which can be collocated by the upper objective, and Olympus uplanApo/IR 60×/1.2 water immersion for the lower objective. (c). Olympus uplanApo/IR $60 \times / 1.2$ water immersion for the upper objective along with the diffraction orders which can be collocated by the upper objective, and Olympus uplanApo/IR 60×/1.2 water immersion for the lower objective.

When the light hits the object it diffract, a single beam of light will split into several different diffraction orders bents at increasing angles from the original incident light. The easiest way to understand this property of light is to consider what happens when a beam of light shines through a pinhole into a dark background: The obtained images look like a negative target with a large central disk of light surrounded by a series of thin circles of light of decreasing brightness as it goes further away from the center. 


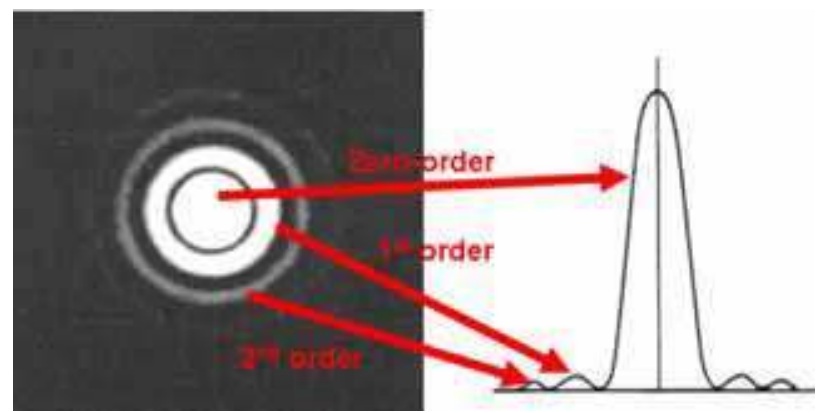

Fig. 15. The diffraction orders which are suppose to be collected by the objectives.

\section{Discussion}

The light-gathering ability of a microscope objective is quantitatively expressed in terms of the numerical aperture, which is a measure of the number of highly diffracted imageforming light rays captured by the objective. Higher values of numerical aperture allow increasingly oblique rays to enter the objective front lens, producing a more highly resolved image. An important concept to understand the image formation is the nature of diffracted light rays intercepted by the objective. Only in cases where the higher (1st, 2nd, 3rd, etc.) orders of diffracted rays are captured, can interference work to recreate the image in the intermediate image plane of the objective (see Figs. 14 and 15). When only the zeroth order rays are captured, it is virtually impossible to reconstitute a recognizable image of the specimen. When first order light rays are added to the zeroth order rays, the image becomes more coherent, but it is still lacking the sufficient detail. It is only when higher order rays are recombined, that the image will represent the true architecture of the specimen. This is the basis for the necessity of large numerical apertures (and subsequent smaller Airy disks, see Fig. 15) to achieve high-resolution images with an optical microscope.

The brightness of an image formed by an objective at a fixed magnification increases with the diameter of the angular aperture (the angle of the cone of light collected by the objective) see Fig. 14. Light rays emanating from the specimen proceed through air (or an immersion medium) that lies between the coverslip and the objective front lens. The angular aperture is expressed as the angle between the microscope optical axis and the direction of the most oblique light rays captured by the objective. The numerical aperture is expressed as:

$$
\text { Numerical Aperture (N.A.) }=\mathrm{n} \cdot \sin \theta
$$

where $\mathrm{n}$ is the refractive index of the medium in the object space (between the coverslip and the objective front lens) and $\theta$ is one-half the angular aperture (see Fig. 14). The value of $n$ varies between 1.0 for air and 1.33 for a majority of immersion water utilized in optical microscopy. The angular aperture, which varies with the objective focal length, is the maximum angle of image-forming light rays emanating from the specimen that the objective front lens can capture when the specimen is focused. As the objective focal length decreases, the maximum angle between the specimen and the outside diameter of the objective front lens increases, causing a proportionate increase in the angular aperture. From equation (2), it is obvious that numerical aperture increases with both angular aperture and the refractive index of the imaging medium. Theoretically, the highest angular aperture obtainable with a 
standard microscope objective would be 180 degrees, resulting in a value of 90 degrees for the half-angle utilized in the numerical aperture equation. The sine of 90 degrees is one, which indicates that numerical aperture is limited not only by the angular aperture, but also by the imaging medium refractive index. A majority of microscope objectives are designed to operate with air (which has a refractive index of 1.0) as the imaging medium between the coverslip and the objective front lens. This yields a theoretical maximum numerical aperture of 1.0, but in actual practice, the highest numerical aperture for a dry objective is about 0.95 (the angular aperture half-angle equals approximately 72 degrees).

The visual field brightness (B) of the microscope is determined by;

$$
\text { B a (N.A. })^{2} /\left(\mathrm{M}_{\text {objective }}\right)^{2}
$$

where $\mathrm{O}_{\text {bjective }}$ is the objective lens magnification. Following equation (3) one can conclude that the brightness will increase as the numerical aperture increases and / or as the objective magnification decreases.

The resolving power of an objective lens is measured by its ability to differentiate two lines or points in an object. As the resolving power increases, the smaller the distance between the two lines or points to be distinguished can be. The more light that is collected by the objective lens the better resolution is and therefore as N.A. increases, so does the resolving power. The resolving power can be determined by:

$$
\text { Resolving Power }=0.61 \cdot \lambda / \text { N.A. }
$$

When the light rays come out of an axial object point enters a lens, the light rays with a larger N.A. are subjected to stronger refraction power and cross the optical axis in positions with larger differences from the ideal image formation position. As a result spherical aberrations are proportional to the N.A. ${ }^{3}$.

The SH signals were obtained in both directions. These signals increased considerably when the chloroplasts rotated upon being illuminated by linearly polarized femtosecond pulses laser of paired photons which are effectively equivalent to blue light at half the wavelength of the irradiating laser (Cox et al., 2004; Reshak et al., 2009; Reshak, 2009). This chromatically equivalent blue range caused chloroplasts to rotate under the linearly polarized pulses laser causing orientation parallel to the side walls of the leaf cells. Starch grains are not involved thus eliminating a possible source of confusion. SHG indicates the non-centrosymmetric nature of the chloroplast membranes especially when aggregated into grana. The chloroplast grana and intergranal membranes are exhibit strong birefringence with large variations (Pantic-Tanner and Eden, 1999; Garab et al., 2005). A low intrinsic birefringence is expected in intergranal membranes and a high birefringence where the membranes are stacked. The grana were expected to give a stronger $\mathrm{SH}$ signal than the intergranal regions with unstacked membranes. During rotation of the chloroplast caused by linearly polarized high light intensity designed to present a smaller interface to the incoming light, the SHG increased considerably compared with the intergranal regions.

\section{Conclusions}

The enhancement of the forward direction of SH imaging was demonstrated by using set of different numerical aperture objectives with different immersion medium. The experiment shows that the numerical aperture is limited not only by the angular aperture, but also by 
the refractive index of the immersion imaging medium. The SHG signals are dependent on the numerical aperture and the refractive index of the immersion imaging medium. The brightness of the image depends on the axial aspect of the chloroplast. The image intensity also depends on the orientation of the chloroplast in relation to the illuminating polarization direction. A light-induced re-orientation in dependence of the illumination intensity could be observed (Reshak et al., 2009; Reshak, 2009).

\section{Rotation of birefringent biological microparticles by linearly polarized laser using multifunctional two-photon laser scanning microscope}

As it has been mentioned above, the chloroplast produced very strong birefringence which is originated from grana and the stacked regions of the thylakoid membranes. The chloroplast grana and intergranal membranes are distinguished by their birefringence (Pantic-Tanner and Eden, 1999). A low intrinsic birefringence is expected in intergranal membranes and a high birefringence where the membranes are stacked. When the incident beam of the linearly polarized light interacts with the chloroplast a considerable amount of torque will generates resulting in movements of the chloroplasts. Since the chloroplasts are non-centrosymmetric particles, measurable amount of second harmonic will generates without photo-damage or photo-belching by utilizing the fact that the SHG is energy conservation process. In this case one can expose the chloroplast to the laser radiations without risk. The intensity distribution of the SHG signals which comes from the chloroplast while illuminated by linearly polarized laser is shown in Fig. 16. Following these images, one can see the intensity distribution of the $\mathrm{SH}$ signal reduces as a consequence of the rotation during the exposure time, as axial thickness increases (when the chloroplast rotate around the short axis the axis in the plane of the paper) the SHG signal will decrease in intensity, thus one can conclude that the chloroplast attempt to rotate and move away from the laser light in order to protect the photosynthetic system from being exposed to high intensity.

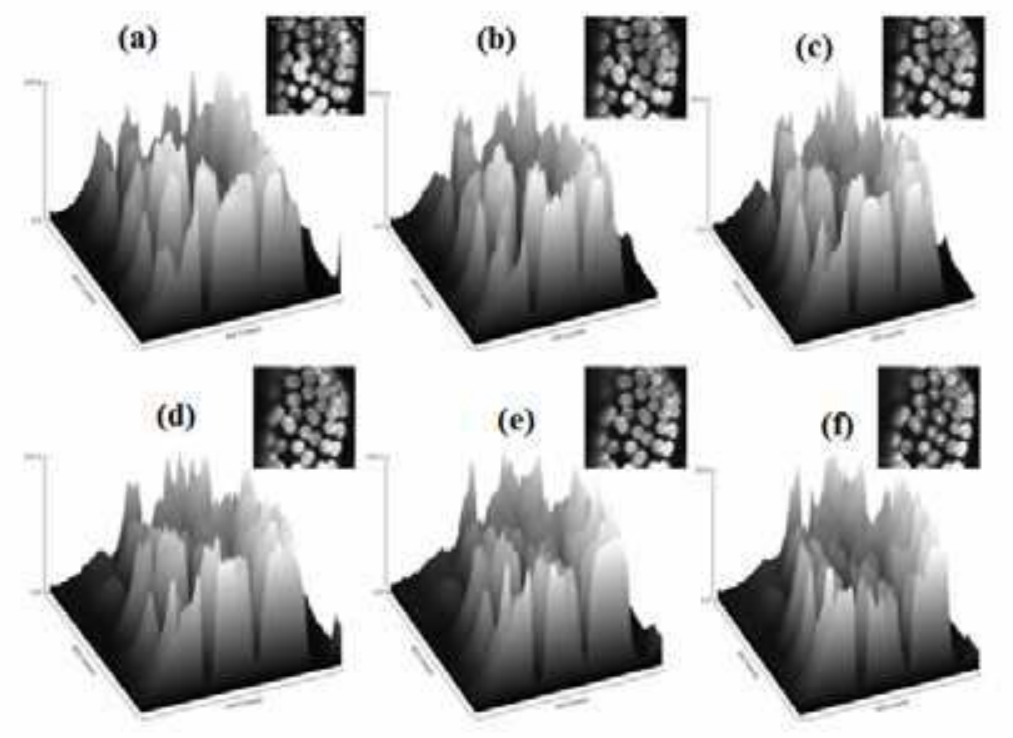

Fig. 16. Intensity distribution of the SH signals during the movements and rotation. 


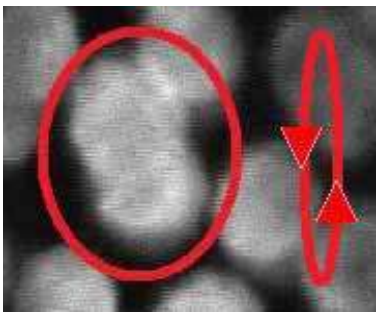

(a)

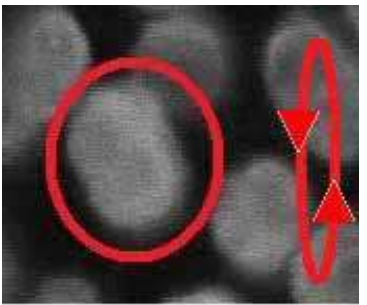

(d)

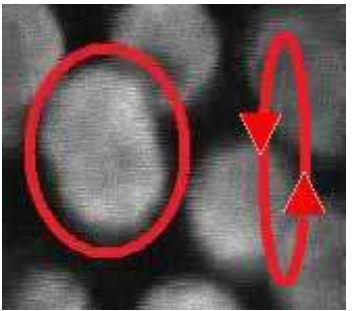

(b)

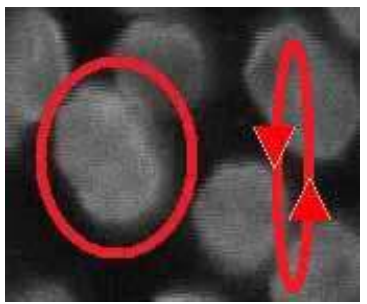

(e)

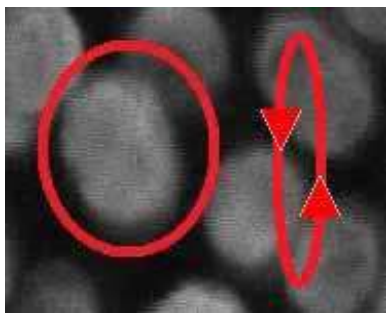

(c)

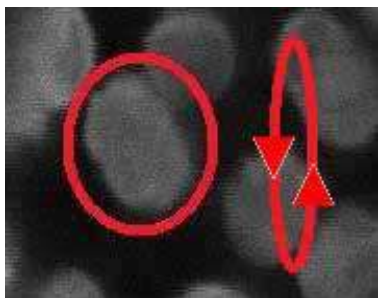

(f)

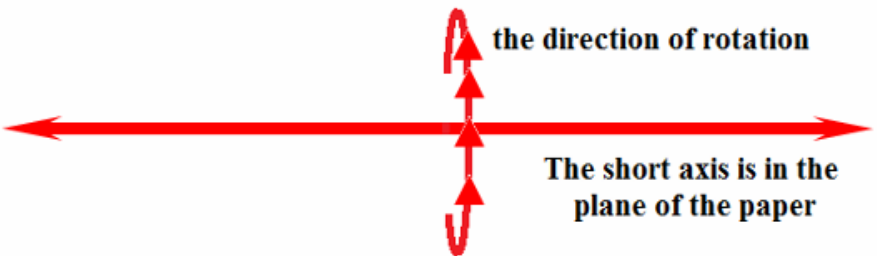

Fig. 17. Selected chloroplast illustrates the rotation around certain axis (axis parallel to the plane of the paper).

Fig. 17, shows the rotation of the chloroplast around certain axis (axis parallel to the plane of the paper), by this rotation of the chloroplast the dipole momentum in side the molecules oriented to be perpendicular to the polarization's direction of the laser beam. The SHG signal of the chloroplasts was collected using the objective Olympus uplanApo/IR 60×/1.2 water immersion. The $\mathrm{SH}$ signal showed no signs of bleaching during acquisition of repeated images from a given area, and thus showing no damage to the structure. This is to be expected since SHG is a coherent process, unlike fluorescence, and no energy is lost (Gorrell, 2003).

\section{Discussion}

Previous studies (Bayoudh et al., 2000) demonstrate that displacement of chloroplast inside the cell is extremely difficult, presumably due to chloroplast adhesion to the cytoskeleton and connections between organelles. In the present study we demonstrate that displacement and rotation of the chloroplast inside the cell were done using linearly polarized laser without photo-damage or photo-belching. For this experiment the setup in Fig. 18 was used. A linearly polarized laser beam was used to induce second harmonic generation signals from the chloroplast in Plagiomnium affine leaves. Since the light can carry angular 


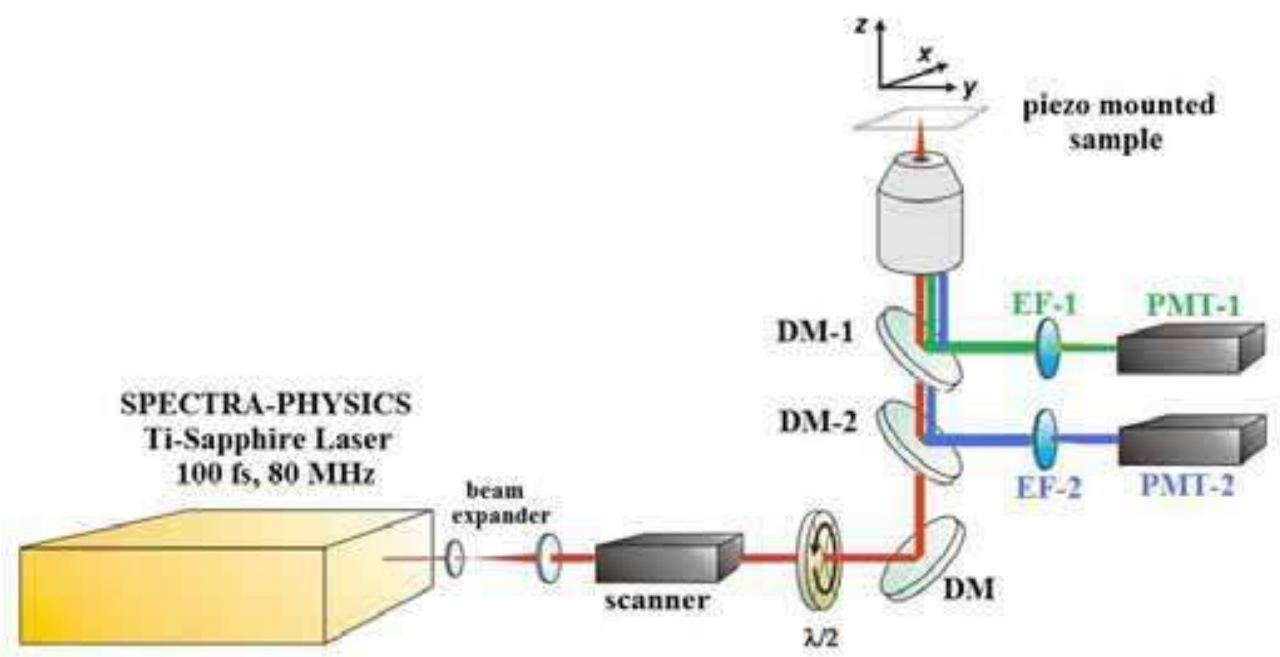

Fig. 18. The experiment the setup

momentum as well as linear momentum, the incident beam of linearly polarized laser becomes elliptically polarized and gains angular momentum after encroaching on a birefringent particle whose optical axis is not parallel to that of polarization of the light (Higurashi et al. 1999; Garab et al., 2005). The resulting change in angular momentum generates a reaction optical torque. Since the chloroplast exhibit a strong birefringence, the resulting anisotropic interaction with the linearly polarized laser beam strongly contributes to the optical torque orienting the chloroplast makes it possible to manufacture micromotors for micromechanical systems. The optical torque can be controlled by changing the laser's power following the formula (Higurashi et al. 1999);

$$
\tau_{\text {linear }}=-\frac{P}{\omega} \sin \left(2 \pi \frac{R}{\lambda}\right) \sin (2 \theta)
$$

where $\tau$ is the optical torque generated by the linearly polarized laser acting on birefringent particle, $P$ is the power of the linearly polarized laser at the particles, $\omega$ and $\lambda$ are the frequency and the wavelength of the linearly polarized laser, respectively, $\theta$ is the angle between the polarization direction and the slow birefringence axis of the particle (the axis along which the index of refraction is largest), to obtain considerable amount of the optical torque the angle $\theta$ should be greater than zero, moreover the optical torque should be strong enough to overcome the Brownian motion, the latter is randomly oriented, and $R$ is the retardation of the particle, defined as $R=\Delta n \times d$, where $\Delta n$ is the birefringence defined as $\Delta n=n_{e}-n_{0}$, and $d$ is the thickness of the particle. From this formula it is clear that laser power and the retardation term play major role in reducing / increasing the optical torque at a certain frequency and wavelength. The contribution of retardation part is come from the property of the particle so one has to select thick particle which possesses large birefringence in order to gain considerable amount for $R$. The laser's power $P$ is one property of the setup meaning that it is under control, and one can increase / reduce the 
power to a certain limit without causing photo-damage or photo-belching to the sample by utilizing the fact that the SHG is known to leave no energy deposition to the interacted matters due to their virtual energy conservation characteristic. Since both $P$ and $R$ are proportional to $\tau$, a particle with large $R$ helps to use less laser's power, however by increasing the laser's power one can gain considerable amount of $\tau$, which eventually overcomes the Brownian motion and the adhesion power of the chloroplast to the cytoskeleton and connections between organelles resulting in increasing the speed of the rotation. When a chloroplast inside a cell was rotate and displaced from its original position the nearby chloroplasts were rotating and displaced as well and followed the nearest neighbor. That is attributed to the fact that the chloroplasts were connected to each other by fine cytoplasmic strands or microfilaments. Thus, in general to maximize the signal short pulses should be used and average laser power should be kept in limit to prevent heating of the sample. We should emphasize that the asymmetric shape will contribute to the optical torque. Optically induced torque is always a result of transfer of angular momentum from light to a particle with conservation of momentum as an underlying principle. Consequently, rotation can be induced by a beam of light that carries angular momentum or by a beam that carries no angular momentum but where angular momentum is induced in the beam by the particle.

It is well known from Maxwell's theory that electromagnetic radiation carries both energy and momentum. The momentum may have both linear and angular contributions; angular momentum has a spin part associated with polarization (Allen et al., 1992) and orbital part associated with spatial distribution (Bijersbergen et al., 1992). Any interaction between radiation and matter is inevitably accompanied by an exchange of momentum. This often has mechanical consequences some of which are related to radiation pressure.

Light can carry angular momentum in two distinct forms; spin angular momentum, associated with the polarization state of the beam and orbital angular momentum, associated with the spatial distribution of the beam (Padgett and Allen, 2000). Optical torque is produced if either of these two forms of angular momentum is altered during the scattering of light.

The optical torque acting on the particle is equal and opposite to the rate of change of the angular momentum of the laser beam as it passes through the particle. Since the chloroplasts are birefringent particles, the angular momentum is transferred to the polarization state of the transmitted beam. Before acting on birefringent particles the linearly polarized light composed of equal quantities of left and right circular polarization, so it contains no net angular momentum. Generating a torque on chloroplasts (birefringent particles) causes an imbalance in the power of the left and right circular components of the transmitted beam, the spin torque acting on the chloroplasts is;

$$
\tau=\left(P_{\text {Right }}-P_{\text {Left }}\right) / \omega_{0}
$$

where $P_{\text {Right }}$ and $P_{\text {Left }}$ are the right and left circularly polarized components, respectively; $\omega_{0}$ is the optical angular frequency. If there is no particles then $P_{\text {Right }}=P_{\text {Left }}$.

This work describes experiments using linearly polarized laser to probe chloroplast. The linearly polarized laser was used to induce motion and tumbling of the chloroplast in cells of living leaf tissue. 


\section{Conclusions}

We have demonstrated the movements and the rotation of the chloroplast of the Plagiomnium affine leaves while being illuminated by linearly polarized laser. Since the chloroplast produced very strong birefringence, the resulting anisotropic interaction with the linearly polarized laser beam strongly contributed to the optical torque orienting the chloroplast which makes it possible to manufacture micromotors for micromechanical systems. The torque can be controlled by changing the laser's power. The laser power and the retardation part play a major rule in reducing / increasing the optical torque at a certain frequency and wavelength. The contribution of retardation part comes from the property of the particle so one has to select thick particle which possess large birefringence in order to gain considerable amount for $R$. The laser's power $P$ is one property of the setup that means it is under control, and one can increase/reduce the power to certain limit without cause photo-damage or photo-belching to the sample by utilizing the fact that the SHG is known to leave no energy deposition to the interacted matters due to their virtual energy conservation characteristic. Since both of $P$ and $R$, proportional to $\tau$, a particle with large $R$ it helps to use less power, however, by increasing the laser's power one can gain considerable amount of $\tau$ which is overcomes to the adhesion power of the chloroplast to the cytoskeleton and connections between organelles resulting in increasing the speed of the rotation.

\section{Acknowledgements}

This work was supported from the institutional research concept of the Institute of Physical Biology, UFB (No.MSM6007665808), the program RDI of the Czech Republic, the project CENAKVA (No. CZ.1.05/2.1.00/01.0024), the grant No. 152/2010/Z of the Grant Agency of the University of South Bohemia. The School of Microelectronic Engineering, University Malaysia Perlis (UniMAP), Block A, Kompleks Pusat Pengajian, 02600 Arau Jejawi, Perlis, Malaysia.

\section{References}

Allen L., Beijersbergen M. W., Spreeuw R.J.C. and Woerdman J. P., Phys. Rev. A 45 (1992) 8185.

Born, M., Wolf, E., 1980. Principle of Optics. Cambrige University Press, Cambrige

Boyd, R., 1992. Nonlinear Optics. Academic, New York.

Bayoudh S., Mehta M., Rubinsztein-Dunlop H., Heckenberg N. R. and Critchley C, Journal of Microscopy, Vol. 203, 214-222 (2000).

Beijersbergen, M. W., Allen, L., van der Veen, H. E. L. O., and Woerdman, J. P., 1993, Opt. Commun., 96, 123

Campagnola P. J., Wei M. D., Lewis A., and Loew L. M., High-resolution nonlinear optical imaging of live cells by second harmonic generation Biophys. J. 77, 3341-3349 (1999).

Campagnola P. J., Clark H.A., Mohler W.A., Lewis A. and Loew L.M., "Second-harmonic Imaging Microscopy of Living Cells,“ J. Biomed. Opt. 6, 277-286 (2001) 
Campagnola PJ, Loew LM. Second-harmonic imaging microscopy for visualizing biomolecular arrays in cells, tissues and organisms., Nat Biotechnol. 2003 Nov;21(11):1356-60.

Cheng J., Volkmer A., and Xie X.S., "Theoretical and experimental characterization of coherent anti-Stokes Raman scattering microscopy" J. Opt. Soc. Am B 19, 1363-1375 (2002).

Cox G., Kable E., Jones A., Fraser I., Manconi F. , and Gorrell M. D., “3Dimensional Imaging of Collagen Using Second Harmonic Generation," J. Struct. Bio., 141, 53-62 (2003)

Cox G., Moreno N., and Feijó J., Second harmonic imaging of plant polysaccharides. Journal of Biomedical Optics 10, 024013_1-6 (2004).

Clowes F.A.L. and Juniper B.E, Plant Cells. Blackwell Scientific Publication, Oxford and Edinburgh. 546pp, 1968.

Chu S., Chen I., Liu T., Chen P. C., Sun C. K., and Lin B. L., Multimodal nonlinear spectral microscopy based on a femtosecond Cr:forsterite laser, Optics Letters, Vol. 26, Issue 23, pp. 1909-1911 (2001).

Cheng J., Volkmer A., and Xie X.S., "Theoretical and experimental characterization of coherent anti-Stokes Raman scattering microscopy" J. Opt. Soc. Am B 19, 1363-1375 (2002).

Denk W., Piston D. W. and Webb W. W., Two-photon molecular excitation in laser scanning microscopy, Handbook of Biological Confocal Microscopy, ed J B Pawley (New York; Plenum) pp 445-58 (1995).

Denk W., Strickler J.H., and Webb W.W., “Two-Photon Laser Scanning Fluoresence Microscopy," Science 248, 73-76 (1990).

Diaspro A., Building a two-photonmicroscope using a laser scanning confocal architecture, in Methods in Cellular imaging, A. Periasamy, Ed., pp. 162-179, Oxford University Press, New York (2001).

Faludi-Daniel A., Bialek G.E., Horvath G., Sz.-Rozsa Z., and Gregory R. P. F., Differential light scattering of granal and agranal chloroplasts and their fragments, Biochem. J. 174, 647-651 (1978).

Frohn J. T., Knapp H. F., and Stemmer A., True optical resolution beyond the Rayleigh limit achieved by standing wave illumination, Proc. Natl Acad. Sci. USA 97, 7232-7236 (2000).

Freund I., Deutsch M., and Sprecher A., "Connective Tissue Polarity, Optical secondharmonic microscopy, crossed-beam summation, and small-angle scattering in rattail tendon," Biophy. J. 50, 693-712 (1986).

Fine S., and Hansen W. P., Optical Second Harmonic Generation in Biological Systems Appl. Opt. 10, 2350-2353 (1971).

Gao L., Jin L. X. P., Xu J. W. Y., Ma Hui, and Chen D., Reconstruction of complementary images in second harmonic generation microscopy, Optics Express 14, 4727-4735 (2006).

Garab G., Galajda P., Pomozi I., Finzi L., Praznovszky T., Ormos P., Van Amerongen H., Alignment of biological microparticles by a polarized laser beam. Eur Biophys J 34, 335-343 (2005). 
Georgiou E., Theodossiou T., Hovhannisyan V., Politopoulos K., Rapti G. S., and Yova D., Second and third optical harmonic generation in type I collagen, by nanosecond laser irradiation over a broad spectral region, Opt. Commun. 176, 253-260 (2000).

Gauderon R., Lukins P. B., and Sheppard C. J. R., Three-dimensional second-harmonic generation imaging with femtosecond laser pulses, Opt. Lett. 23, 1209-1211 (1998).

Goedheer JC. Orientation of the pigment molecules in the chloroplast. Biochim Biophys Acta 16:471-476 (1955).

Gorrell, M.D., Wang, X.M., Levy, M.T., Kable, E., Marinos, G., Cox, G., McCaughan, G.W., 2003. Interahepatic expression of collagen and fibroblast activation protein (FAP) in hepatitis C virus infection. Adv. Exp. Med. Biol. 524, 235-243.

Haupt W. And Scheuerlein R., Chloroplast movement. Plant Cell Environm 13, 595-614 (1990).

Han M., Giese G., and Bille J. F., Second harmonic geration imaging of collagen fibrils in cornea and sclera, Optics Express 13, No.15, 5791-5796 (2005).

Heinz, T.F., Chen, C.K., Ricard, D., Shen, Y.R., 1982. Phys. Rev. Lett. 48, 478-481.

Helmchen F. and Denk W., Deep tissue two-photon microscopy, Nature Methods 2, No.12, 932-940 (2005).

Higurashi E., Sawada R., and Ito T., Optically induced angular alignment of trapped birefringent micro-objects by linearly polarized light. Phys. Rev. E 59:3676-3681 (1999)

(http://www.till-photonics.com/Products/imic.php)

Lukins P. B., Xu P., Rehman S. and Sheppard C.J.R., Comparsion of two photon optical microscopies: second harmonic generation and fluorescence imaging, Science, Technology and Eduction of Microscopy: an overview, Volume 2, A Mendez-Vilas, editor, Kluwer/Formatex, Badajoz, ISBN 84-607-6699-3, PP. 712-719 (2003)

Maiti S., Shear J. B., Williams R. M., Zipfel W. R. and Webb W. W., “Measuring serotonin distribution in live cells with three-photon excitation," Science 275, 530-532 (1997).

Mark D. Gorrell, Xin M. Wang, Miriam T. Levy, Eleanor Kable, G. Marinos, Guy Cox, and G. W. McCaughan., Interahepatic expression of collagen and fibroblast activation protein (FAP) in hepatitis $C$ virus infection, Advances in Experimental Medicine and Biology 524, 235-243 (2003)

Mertz J., and Moreaux L., "Second harmonic generation by focused excitation of inhomogeneously distributed scatterers", Opt. Commun. 196, 325-330 (2001).

Mason W. T. , Fluorescent and Luminescent Probes for Biological Activity, second Edition, Academic Press, London (1999).

Moreaux L., Sandre O., and Mertz J., "Membrane imaging by second harmonic genration microscopy“ J. Opt. Soc. Am B 17, 1685-1694 (2000)

Moreno N., Feijo J., and Cox G., Implementation and evaluation of a detector for forward propagated second harmonic signals, Micron, 35, 721-724 (2004).

Pantic-Tanner Z. , and Eden Don, "calculation of protein form Birefringence using the finite element method" Biophysical Journal 76, 2943-2950 (1999).

Padgett M, Allen L (2000). Light witha twist in its tail, Contemp. Phys. 41:5, 275-285.

Reshak A. H., Sarafis V., Heintzmann R., Second harmonic imaging of chloroplasts using the two-photon laser scanning microscope, Micron 40, 378-385 (2009). 
Reshak A. H., Second harmonic generation from thick leaves using the two-photon laser scanning microscope, Micron 40, 455-462 (2009).

Reshak A. H., High second harmonic generation signal from muscles and fasciapig's muscles using the two-photon laser scanning microscope, Journal of Microscopy, Vol. 234, 280-286 (2009).

Reshak A. H., Enhancing the resolution of the forward Second harmonic imaging using the Two-Photon Laser Scanning Microscope, Micron 40, 750-755 (2009).

Reshak A. H. and Auluck S., The linear and nonlinear optical properties of $\mathrm{WS}_{\mathrm{x}} \mathrm{Se}_{2-\mathrm{x}}(\mathrm{x}=0.5$, 1.5, and 2.0) Physica B 393, 88-93 (2007).

Rezende-Pinto, M. C., On the birefringency of chloroplast in higher plants: convergence of results found by the use of the polarizing, light and electron microscopes. Port. Biol. Series A. 12, 267-270 (1972).

Roth S. and Freund I.,"Coherent Optical Harmonic Generation in Rat-tail," Opt. Commun. 33, 292-296 (1980).

Roth S., and Freund I., Imaging cells and extracellular matrix in vivo by using secondharmonic generation and two-photon excited fluorescence Opt. Commun. 33, 292296 (1980).

Sarafis V., Chloroplasts: A structural approach. J Plant Physiol, 152(2-3), 248-264 (1998).

Schrader M., Bahlmann K. and Hell S. W., "Three-photon-excitation microscopy: theory, experiment and applications," Optik 104, 116-124 (1997).

Shen, Y. R. Surface properties probed by second-harmonic and sum-frequency generation, Nature 337, 519 (1989).

Wildman, S.G., Charlene, A.J. \& Atchison, B. A., Light microscopic analysis of the threedimensional structure of higher plant chloroplasts, position of starch grains and probable spiral arrangement of stroma lamellae and grana. Bot. Gaz. 141. 24-36 (1980).

Williams R.M., Zipfel W.R. and Webb W.W., " Interpreting second-harmonic generation images of collagen I fibrils" Biophy. J. 88, 1377-1386 (2005)

Wokosin D. L., Centonze V. E., Crittenden S. and White J., "Three-photon excitation fluorescence imaging of biological specimens using an all-solid-state laser," Bioimaging 4, 208-214 (1996).

Yan, P.; Millard, A. C.; Wei, M.-D.; Loew, L. M. "Unique Contrast Patterns from ResonanceEnhanced Chiral SHG of Cell Membranes" J. Am. Chem. Soc. 128, 11030 (2006).

Yelin D., and Silberberg Y., Laser scanning third harmonic generation microscopy in biology, Optics Express 5, No.8, 169 (1999).

Yeh A.T., Nassif N., Zoumi A. and Tromberg B.J., "Selective corneal imaging using combined second-harmonic generation and two-photon excited f luorescence," Opt. Lett. 27, 2082-2084 (2002)

Zipfel W.R., Williams R.M., and Webb W.W., “Nonlinear magic: multiphoton microscopy in the biosciences,"Nature Biotech. 21, 1369-1377 (2003).

Zumbusch A., Holtom G.R., and Xie X.S., "Three-Dimensional Vibrational Imaging by Coherent Anti-Stokes Raman Scattering," Phys. Rev. Lett. 82, 4142-4145 (1999). 
Zoumi A., Yeh A., and Tromberg B. J., Imaging cells and extracellular matrix in vivo by using second harmonic generation and two photon excited fluorescence, PNAS 99, 11014-11019 (2002). 


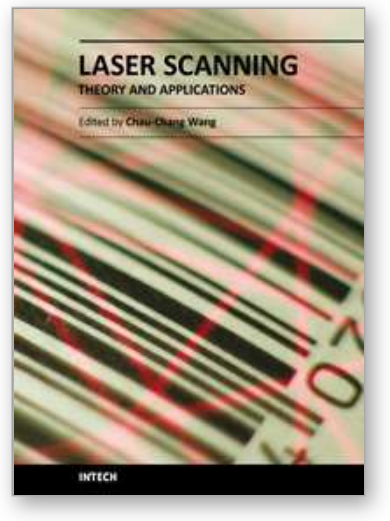

\author{
Laser Scanning, Theory and Applications \\ Edited by Prof. Chau-Chang Wang
}

ISBN 978-953-307-205-0

Hard cover, 566 pages

Publisher InTech

Published online 26, April, 2011

Published in print edition April, 2011

Ever since the invention of laser by Schawlow and Townes in 1958, various innovative ideas of laser-based applications emerge very year. At the same time, scientists and engineers keep on improving laser's power density, size, and cost which patch up the gap between theories and implementations. More importantly, our everyday life is changed and influenced by lasers even though we may not be fully aware of its existence. For example, it is there in cross-continent phone calls, price tag scanning in supermarkets, pointers in the classrooms, printers in the offices, accurate metal cutting in machine shops, etc. In this volume, we focus the recent developments related to laser scanning, a very powerful technique used in features detection and measurement. We invited researchers who do fundamental works in laser scanning theories or apply the principles of laser scanning to tackle problems encountered in medicine, geodesic survey, biology and archaeology. Twenty-eight chapters contributed by authors around the world to constitute this comprehensive book.

\title{
How to reference
}

In order to correctly reference this scholarly work, feel free to copy and paste the following:

Ali Hussain Reshak (2011). Second Harmonic Generation Signal from Biological Materials Using MultiFunctional Two-Photon Laser Scanning Microscopy, Laser Scanning, Theory and Applications, Prof. ChauChang Wang (Ed.), ISBN: 978-953-307-205-0, InTech, Available from:

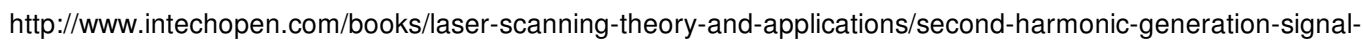
from-biological-materials-using-multi-functional-two-photon-laser-

\section{INTECH}

open science | open minds

\section{InTech Europe}

University Campus STeP Ri

Slavka Krautzeka 83/A

51000 Rijeka, Croatia

Phone: +385 (51) 770447

Fax: +385 (51) 686166

www.intechopen.com

\section{InTech China}

Unit 405, Office Block, Hotel Equatorial Shanghai

No.65, Yan An Road (West), Shanghai, 200040, China 中国上海市延安西路65号上海国际贵都大饭店办公楼 405 单元

Phone: +86-21-62489820

Fax: $+86-21-62489821$ 
(C) 2011 The Author(s). Licensee IntechOpen. This chapter is distributed under the terms of the Creative Commons Attribution-NonCommercialShareAlike-3.0 License, which permits use, distribution and reproduction for non-commercial purposes, provided the original is properly cited and derivative works building on this content are distributed under the same license. 